\title{
Impacto del mindfulness en el rendimiento académico de estudiantes universitarios: una revisión sistemática de literatura con VOSviewer
}

The impact of mindfulness on university students' academic achievement: a systematic literature review with VOSviewer

Recibido: 16 de noviembre 2020, evaluado: 20 de febrero 2021, aceptado: 10 de abril 2021

\author{
Alfredo José Galdo Jiménez \\ galdoalfredo@gmail.com \\ https://orcid.org/0000-0002-9795-7524 \\ Universidad de Lima-Perú \\ R e s u m e n
}

La presente investigación tiene por objetivos determinar el nivel de impacto de la exclusión de algunos términos claves en la coocurrencia acerca del tema "Impacto del mindfulness en el rendimiento académico de estudiantes universitarios", así como determinar los países con el mayor ratio citaciones/ documento y precisar el nivel de impacto de la reducción de cantidad de documentos en 1 y aumento en la cantidad de citaciones en 4 en la determinación de los autores con mayor ratio citación/ documento. El diseño de estudio de revisión sistemática es de tipo transversal descriptivo, utilizando muestreo no probabilístico de tipo intencional. La muestra tiene 51 documentos extraídos de la base de datos Scopus del periodo 2015-2020 y que coincidan con la ecuación de búsqueda recogida del tema de investigación. El instrumento para el análisis es el software VOSviewer. Los resultados muestran que las palabras claves mindfulness y academic achievement son las de mayor impacto y que sería recomendable ahondar en publicaciones relacionadas con la psicología. Otro resultado de la investigación indica que se debe considerar como prioridad a Estados Unidos, Alemania y Australia por tener las ratios más altas de citación/documento. Finalmente, se concluye que la reducción de cantidad de documentos en 1 y aumento en la cantidad de citaciones en 4 tiene impacto en la determinación de los autores con mayor ratio citación/documento, recomendándose a los autores Hahne A., Mccaleer R. y Mcconville J, por ser quienes tienen más altos ratios citación/documento, además de publicaciones relativamente recientes.

Palabras clave: nivel de impacto, términos clave, coocurrencia, ratio, citación 


\section{Summary}

The current research study objectives are focused on determine in first place the level impact of the exclusion of some key terms on the co-occurrence variable about the research topic "The impact of mindfulness on university students' academic achievement"; in second place, determine countries with the highest citation per document rate and finally define the impact level of the reduction of the amount of documents and the increase of the amount of citations on the determination of the authors with the highest citation- per document- rate. Study design: Sistematic review with cross-sectional analysis and descriptive method. It is used an intentional and non-probabilistic sampling technique. The sample is conformed by 51 documents chosen from Scopus database for the period 2015-2020 and related to the research study equation. For the analysis it is used VOSviewer software. The results obtained shew that due to the fact the words: mindfulness and academic achievement have the highest impact; it would be advisable to deepen on publications related to ychology. In adittion, it must be a priority to consider United States of America, Germany and Australia publications because of their higher citation-per-document rate. Finally, it is concluded that the reduction of the amount of documents by one and the increase of the amount of citations by four have an impact on the determination of the authors with the highest citation-per-document rate. Hahne A., Mccaleer R. and Mcconville are the recommended authors because of their highest citationper-document rate and their relatively recent publications. ps

Key words: impact level, key terms, co-ocurrence, rate, citation

\section{Introducción}

Hoy en día la docencia ha adquirido otro giro. Las nuevas formas de enfocar el proceso de aprendizaje se sostienen en estudios sobre el aprendizaje cognitivo y la convergencia de teorías sobre el contexto y naturaleza del aprendizaje. Estas teorías indican que los factores psicológicos cobran vital importancia. Y dentro de estos factores se encuentran la motivación, percepción y actitudes. Así, en lugar de enseñar, los docentes deben preocuparse por crear condiciones para el aprendizaje que les permita a los estudiantes no solamente desarrollar habilidades y aprender contenidos, sino desarrollar actitudes favorables para aprender. "Normalmente, el profesor sabe qué hay que hacer, -puede saberlo en teoría-, pero hay una 
gran distancia entre la teoría y la práctica; la gran cuestión es ¿cómo hacer lo que hay que hacer?" (Javier, 2013).

Las actitudes favorables respecto al aprendizaje se establecen si se trabaja sobre lo que ocurre en el plano psicológico y es que los estudiantes en edad universitaria se enfrentan a demandas muy intensas. Pueden consumir energía en recuerdos como también en pensamientos sobre un futuro que aún no llega y si llega no saben cómo será su devenir y su capacidad de afrontarlo (Redero-García, 2016). De otro lado, el estrés, la ansiedad, el tiempo de sueño, así como la baja capacidad de resiliencia pueden jugar en contra para su rendimiento académico.

Es en este contexto que cobra una vital importancia el mindfulness. Kadam-Zinn (2009) indica que este término tiene sus raíces en el budismo y es que etimológicamente el término proviene de Sati, una palabra en lengua Pali usada en la redacción de antiguos textos de budismo y que guarda vinculación al entrenamiento mental, a la atención o conciencia (Redero-García, 2016). El mindfulness en estos tiempos se constituye en "un despertar de la conciencia y un desarrollo personal, que nos lleva al equilibrio y tranquilidad con nosotros mismos y con el resto del mundo" (Redero-García, 2016). Sin embargo, este equilibrio y tranquilidad no está exento de considerar otros factores importantes, como el rol que juega la familia y la salud emocional del docente.

En sus inicios el mindfulness fue aplicado en 1978 por Kadam-Zimm al estrés a través del programa Mindfulness Basado en la Reducción del Estrés (MBSR). Y es desde esa fecha que las investigaciones sobre esto y sus áreas de aplicación han tenido un crecimiento sostenido, lo que hace que el mindfulness se aplique actualmente no solamente en el campo de la salud a partir de intervenciones en hospitales y clínicas, sino también a todo lugar donde existan personas y comunidades (Moñivas et al., 2012). Siendo dentro de estas comunidades que se encuentra la comunidad estudiantil universitaria, sobre la que se centra el presente tema de investigación: Impacto del mindfulness en el rendimiento académico de estudiantes universitarios.

Sin embargo, para comprender y delimitar la estructura del conocimiento, se debe partir de análisis de producción indexada en bases de datos para identificar líneas de investigación y sus interconexiones. Es en este sentido que la revisión sistemática con el uso de la herramienta de software VOSviewer permitirá el procesamiento automático de diversas unidades de información contenidas en registros bibliográficos (metadatos) de la base de datos Scopus, lo que ayudará así en la identificación de documentos vinculados que compartan patrones sobre el presente tema de estudio. Los mapas bibliométricos proporcionarán entonces un útil 
entendimiento de la estructura de un dominio científico por medio de representaciones gráficas de unidades de análisis relevantes (Revista et al., 2016) tales como coocurrencia o coautoría por países y autores. El primero de ellos midiendo similitud documental a través de la detección de ocurrencia de palabras clave y el segundo midiendo similitud de coautoría a nivel de países y autores.

Los objetivos son los fines de la investigación, que están fundamentados en la introducción. "No son más que la expresión concreta de los conocimientos que se pretenden alcanzar con la investigación" (Paneque y Habana, 1998). Para este estudio se plantean objetivos generales y también específicos, siendo estos últimos más concretos y los que indican "conocimientos de menor complejidad que se desean alcanzar, los cuales se irán obteniendo durante la investigación y que contribuyen a lograr el objetivo general" (Salgado, 2018).

Objetivo general 1

Determinar el nivel de impacto de la exclusión de algunos términos claves en la coocurrencia del tema "Impacto del mindfulness en el rendimiento académico de estudiantes universitarios" con un mínimo de ocurrencia de 2 palabras claves en los documentos relacionados extraídos de la base de datos Scopus y que corresponden al periodo 2015-2020.

\section{. Objetivo específico 1.1}

Determinar las palabras con mayor coocurrencia en todas las palabras claves del tema "Impacto del mindfulness en el rendimiento académico de estudiantes universitarios" en los documentos relacionados extraídos de la base de datos Scopus y que corresponden al periodo 2015-2020.

\section{Objetivo específico 1.2}

Determinar las palabras con mayor coocurrencia en todas las palabras claves del tema "Impacto del mindfulness en el rendimiento académico de estudiantes universitarios" excluyendo términos clave menos significativos en los documentos relacionados extraídos de la base de datos Scopus y que corresponden al periodo 2015-2020.

Objetivo general 2

Determinar los principales países para la elección de documentos sobre el tema "Impacto del mindfulness en el rendimiento académico de estudiantes universitarios" extraídos de la base de datos Scopus y que corresponden al periodo 2015-2020.

Objetivo específico 2.1 
Determinar los países con el más alto ratio citaciones/documentos del tema "Impacto del mindfulness en el rendimiento académico de estudiantes universitarios" en los documentos relacionados extraídos de la base de datos Scopus y que corresponden al periodo 2015-2020 Objetivo específico 2.2

Determinar los países más actualizados en investigación sobre el tema "Impacto del mindfulness en el rendimiento académico de estudiantes universitarios" a partir de los documentos relacionados extraídos de la base de datos Scopus y que corresponden al periodo 2015-2020.

Objetivo general 3

Precisar si existe impacto de la reducción de cantidad de documentos en 1 y aumento en la cantidad de citaciones por autor en 4 como parámetros de filtro del tema "Impacto del mindfulness en el rendimiento académico de estudiantes universitarios" sobre el ratio citaciones/documentos por autor en los documentos relacionados extraídos de la base de datos Scopus y que corresponden al periodo 2015-2020.

Objetivo específico 3.1

Establecer los autores con el más alto ratio citaciones/documentos por autor del tema "Impacto del mindfulness en el rendimiento académico de estudiantes universitarios" bajo el escenario de un mínimo de 2 documentos por autor y 1 citación por autor en los documentos relacionados extraídos de la base de datos Scopus y que corresponden al periodo 2015-2020.

Objetivo específico 3.2

Establecer los autores con el más alto ratio citaciones/documentos por autor del tema "Impacto del mindfulness en el rendimiento académico de estudiantes universitarios" bajo el escenario de un mínimo de 1 documento y 5 citaciones por autor en los documentos relacionados extraídos de la base de datos Scopus y que corresponden al periodo 2015-2020.

\section{Tipo de investigación}

\section{Según el proceso formal}

Es de tipo inductivo, porque se analizan casos particulares a partir de los cuales se extraen conclusiones generales (Martín, 2015). Para esta investigación, al estudiar una muestra de documentos por países, se concluirá sobre en cuáles profundizar el estudio a partir del ratio de coocurrencia de términos claves.

\section{Según el grado de abstracción}

Es investigación aplicada, porque se centra en "la búsqueda de solución de problemas prácticos y el control de situaciones de la vida cotidiana" (Vargas Cordero, 2009). Al respecto, 
el problema práctico es orientar la búsqueda de documentos de revistas científicas que recojan estudios para el tema de investigación referido al mindfulness y su impacto en el desempeño académico de estudiantes universitarios.

\section{Según el grado de generalización}

Es de investigación-acción, porque "es una investigación aplicada, orientada a decisiones" (Salgado, 2018). En este caso las decisiones están referidas a elegir; por ejemplo, autores y países respecto a publicaciones referidas al mindfulness en el rendimiento académico de estudiantes universitarios.

\section{Según la naturaleza de los datos}

Es cuali-cuantitativa, porque utiliza la revisión sistemática (cualitativa) y por otro lado analiza la relación entre variables numéricas (Fernández \& Díaz, 2003), tales como ratios de citaciones por documentos/autor y citaciones por documentos/países.

\section{Según la concepción del fenómeno educativo}

Es idiográfica, porque estudia los casos particulares; en este caso literatura científica de Scopus para luego describir (Qualitative \& In, 2006). Es a su vez nomotética, porque realiza el registro de una o varias variables a partir de documentos para hacerse generalizaciones, sobre por ejemplo qué autores preferir en la literatura científica sobre el tema (Muñiz, 1994).

\section{Según la orientación}

Es de investigación orientada a decisiones, en este caso, decidir sobre literatura científica a priorizar para el estudio.

\section{Según la manipulación de variables}

Es investigación descriptiva, ya que no pretende "manipular ninguna variable; por el contrario, se limita a observar y describir los fenómenos. La investigación descriptiva puede utilizar metodología cuantitativa o cualitativa" (Salgado, 2018). Asimismo, en este tipo de investigación solo se pretende recoger información sobre los objetos de estudio, sin establecer relaciones entre ellos (Hernández et al., 2014, p, 92).

Si bien es cierto en este estudio se han modificado escenarios; como por ejemplo para el objetivo 3, esto no se hizo para manipular variables independientes como cantidad de citaciones mínimas por autor que generen resultados de búsqueda que más convengan, ya que se estaría afectando la validez de la revisión sistemática a través del sesgo (Urrutia \& Bonfill, 2010). Lo que buscó el estudio en cambio, fue describir cuáles son los países o los autores más relevantes para el tema de investigación y ello es básicamente descriptivo, por ejemplo.

\section{Según la dimensión cronológica}


Es una investigación descriptiva, situada en una base de conocimientos más sólida que los estudios exploratorios (Paneque \& Habana, 1998), encargándose de describir fenómenos.

\section{Según el enfoque}

Es una investigación experimental con variables independientes manipuladas por el investigador (Cortés Cortés \& Iglesias León, 2004). Por ejemplo, al hacer el cambio de escenarios (disminuyendo el número mínimo de documentos por autor y aumentando la cantidad mínima de citas) se quiere determinar el impacto en el ratio de citaciones/documentos por autor.

\section{Según las fuentes}

Es una investigación bibliográfica, donde el propósito es la revisión de literatura. En la actualidad, ante mucha información científica que crece de manera vertiginosa se persigue la obtención eficiente de la información que sea más relevante para el estudio (Sabour, 2017).

\section{Según el lugar}

Es una investigación de campo y de laboratorio, ya que tiene intervención experimental en variables (cantidad de documentos y cantidad de citaciones) y al mismo tiempo recoge información sin intervención, como cuando arroja las estadísticas de Scopus (Salgado, 2018).

\section{Según los datos con los que se trabaja}

Se trata de una investigación primaria porque es un estudio que busca averiguar sobre algo que no se conoce (Aguilera Eguía, 2014). Esto que no se conoce es lo que corresponde a los objetivos descritos para la presente investigación.

En este trabajo no se está abordando información recabada con anterioridad, ya que para la obtención de datos primarios tales como qué país es el que más documentos ha producido respecto a mindfulness y su impacto en el rendimiento académico, se está trabajando con el software VOSviewer.

\section{Según la temporalidad}

Es una investigación transversal en tanto se realizó en el lapso de las tres primeras semanas del mes de octubre del 2020, es decir se corresponde con un estudio con corte en el tiempo (Muñiz, 1994).

\section{Según el número de individuos}

Es una investigación de grupos de "muestras grandes que han sido seleccionadas por algún método de muestreo, probabilístico o no" (Salgado, 2018). Las muestras grandes para este estudio son los 51 documentos obtenidos de la ecuación de búsqueda de Scopus. 
Asimismo, la investigación es de tipo cualitativa (en cuanto a la revisión sistemática) (Urrutia y Bonfill, 2010).

Es de tipo cuantitativa porque se recogen y analizan datos cuantitativos; por ejemplo, cantidad de citaciones de autores y cantidad de documentos por países (Cadena Iñiguez et al., 2017). La investigación cuantitativa se diferencia de la cualitativa "en el uso de técnicas estadísticas para combinar "numéricamente" los resultados frente a un estimador puntual, también denominado "metaanálisis"” (Aguilera Eguía, 2014) y se utilizó la Revisión Sistemática (RS). En este tipo de revisión el centro está en recopilar estudios realizados sobre el tema de investigación partiendo de recursos electrónicos, por ejemplo, la base de datos Scopus (Aguilera Eguía, 2014).

De acuerdo con los aspectos conceptuales novedosos de PRISMA, los juicios y decisiones no están influidos por resultados incluidos en la revisión y ello se ve favorecido al establecer el protocolo de búsqueda previamente a la revisión, pero esto no es óbice para que los criterios de búsqueda pudieran de alguna manera ser modificados a posteriori por razones justificadas (Urrutia y Bonfill, 2010).

Al trabajar con una base de datos como Scopus se considera que hay validez en los estudios de metaanálisis para la revisión sistemática, como también en los resultados. Como bien indican los autores de PRISMA más que calidad (sujeta probablemente a sesgos por el investigador) se busca validez (Urrutia \& Bonfill, 2010).

De otro lado, según los criterios de PRISMA se ha buscado incorporar todos los estudios que se han considerado relevantes para el tema de revisión. Si bien no se ha obtenido la información completa de los estudios ni de los resultados, esto no afecta la validez de la revisión, ya que el presente trabajo no se está abocando a la revisión de los estudios propiamente (Urrutia y Bonfill, 2010).

En cuanto al diseño el presente estudio corresponde a un diseño de tipo transversal descriptivo. Es transversal porque se realiza en un horizonte de tiempo relativamente corto (las tres primeras semanas de octubre) y es descriptivo porque se encarga de describir lo siguiente:

Del objetivo específico 1 del objetivo general 1

Palabras con mayor coocurrencia en todas las palabras claves del tema "Impacto del mindfulness en el rendimiento académico universitario" en los documentos relacionados extraídos de la base de datos Scopus correspondientes al periodo 2015-2020.

Del objetivo específico 2 del objetivo general 1 
Palabras con mayor coocurrencia en todas las palabras claves del tema "Impacto del mindfulness en el rendimiento académico universitario" excluyendo términos clave menos significativos en los documentos relacionados extraídos de la base de datos Scopus correspondientes al periodo 2015-2020.

Del objetivo específico 1 del objetivo general 2

Países con el más alto ratio citaciones/documentos por ciudad del tema "Impacto del mindfulness en el rendimiento académico universitario" en los documentos relacionados extraídos de la base de datos Scopus correspondientes al periodo 2015-2020.

Del objetivo específico 1 del objetivo general 3

Autores con el más alto ratio citaciones/documentos por autor del tema "Impacto del mindfulness en el rendimiento académico universitario" bajo el escenario de un mínimo de 2 documentos por autor y 1 citación de autor en los documentos relacionados extraídos de la base de datos Scopus correspondientes al periodo 2015-2020.

(Del objetivo específico 2 del objetivo general 3)

Autores con el más alto ratio citaciones/documentos por autor del tema "Impacto del mindfulness en el rendimiento académico universitario" bajo el escenario de un mínimo de 1 documento y 5 citaciones por autor en los documentos relacionados extraídos de la base de datos Scopus correspondientes al periodo 2015-2020.

El plan de investigación comprende lo siguiente:

Para el objetivo específico 1 y 2 del objetivo general 1

Análisis de visualización por superposición: con mínimo de ocurrencia de 2 palabras claves. Análisis de visualización de red: con mínimo de ocurrencia de 2 palabras claves.

Análisis de visualización por densidad: con mínimo de ocurrencia de 2 palabras claves.

Para el objetivo específico 1 del objetivo general 2

Análisis de ratio citaciones/documentos por ciudad: 1 como mínimo número de citaciones de un país y 3 como mínimo número de documentos.

Análisis de visualización de red: 1 como mínimo número de citaciones de un país y 3 como mínimo número de documentos.

Análisis de visualización por densidad: 1 como mínimo número de citaciones de un país y 3 como mínimo número de documentos. 
Para el objetivo específico 2 del objetivo general 2

Análisis de visualización por superposición: 1 como mínimo número de citaciones de un país y 3 como mínimo número de documentos.

Para el objetivo específico 1 del objetivo general 3

Análisis de ratio citaciones/documentos por autor (primer escenario): mínimo de 2 documentos de un autor y un mínimo de 1 citación de autor.

Análisis de visualización por superposición (primer escenario): mínimo de 2 documentos de un autor y un mínimo de 1 citación de autor.

Para el objetivo específico 2 del objetivo general 3:

Análisis de citaciones y documentos (segundo escenario): mínimo 1 documento por autor y 5 citaciones como mínimo.

Análisis de visualización de red (segundo escenario): mínimo 1 documento por autor y 5 citaciones como mínimo.

Análisis de visualización por densidad (segundo escenario): mínimo 1 documento por autor y 5 citaciones como mínimo.

Análisis de visualización por superposición (segundo escenario): mínimo 1 documento por autor y 5 citaciones como mínimo.

\section{Base de datos}

Una base de datos se constituye en el método preferido para el almacenamiento estructurado de datos (Maegraith, 1968) y para esta investigación la que se ha consultado es SCOPUS, la que se constituye en "la mayor base de datos de resúmenes y citas de la literatura revisada por pares" (Scopus - Research Intelligence, 2019) y que resulta muy relevante en cuanto se encarga de proporcionar todos los metadatos entregados por los editores.

Los metadatos que incluye Scopus son: “autor (es), afiliación (es), título del documento, año, identificación eléctrica (EID), título fuente, volumen / número / páginas, recuento de citas, fuente, tipo de documento e identificador de objeto digital (DOI)" (Scopus-Research Intelligence, 2019).

Con Scopus se puede obtener análisis sobre documentos por años de publicaciones y de esta manera se observa que la mayor cantidad de documentos son del 2020, lo cual indica existencia de investigación actual sobre el tema (15 documentos). Ver figura 1. 


\section{Figura 1}

\section{Documentos por año}

Documents by year

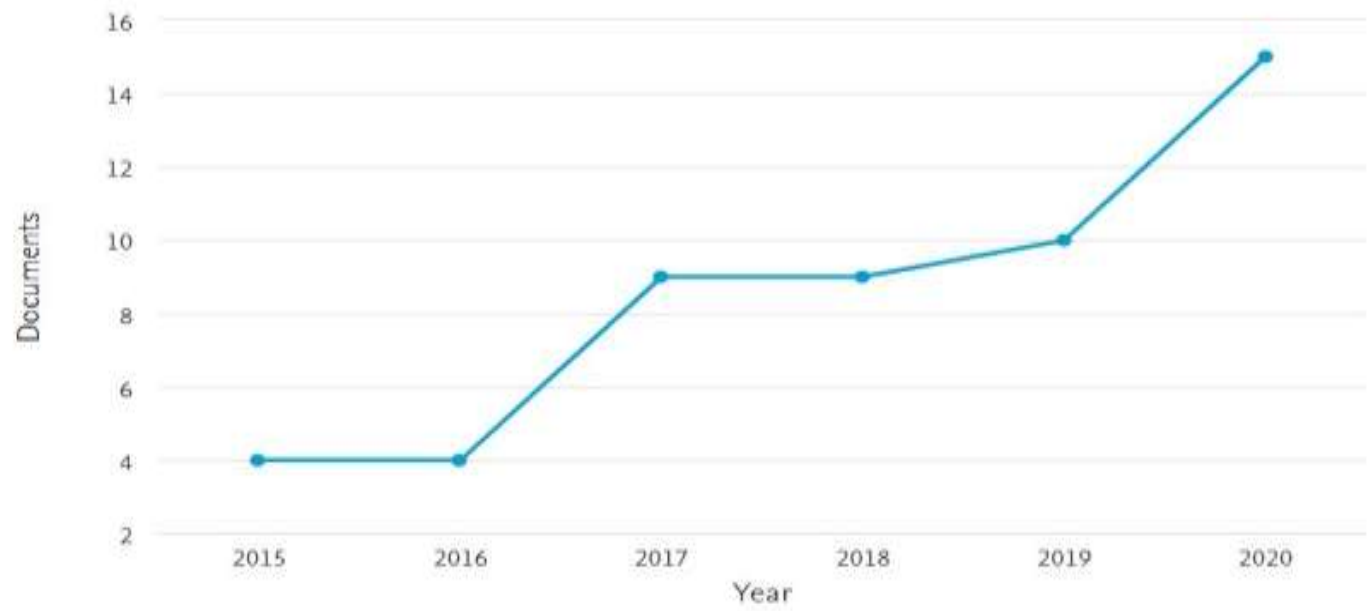

Nota: cantidad de publicaciones anuales de artículos científicos referidos al tema de investigación "Impacto del mindfulness en el rendimiento académico de estudiantes universitarios" extraídos de la base de datos Scopus en el periodo 2015-2020. Recuperado en 17 de octubre de 2020 de https://www-scopus-com

Otro análisis realizado es cantidad de documentos por autor, donde se aprecia que Cuijpers es a quien le corresponde la mayor cantidad junto con Dufour, Ebert, Galante, con dos documentos para cada caso como se aprecia en la figura 2.

\section{Figura 2}

Documentos por autor

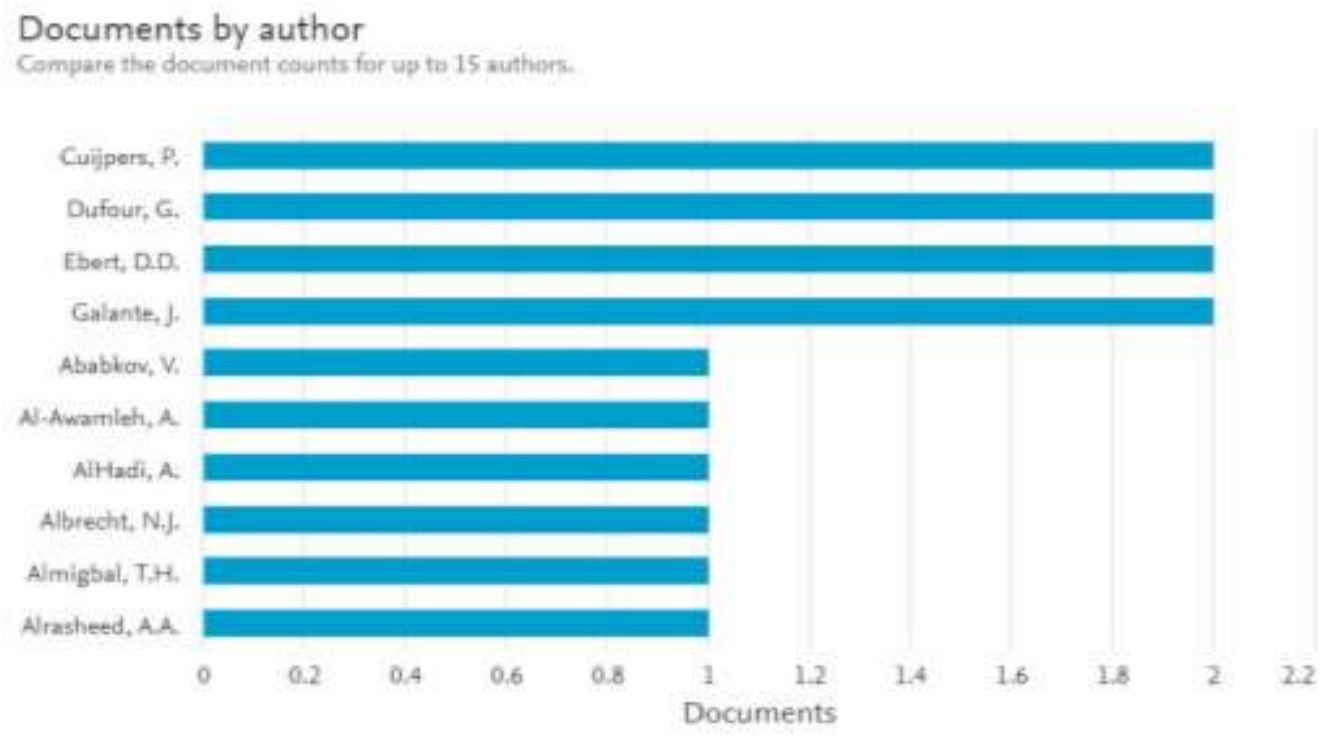

Nota: cantidad de documentos referidos al tema de investigación "Impacto del mindfulness en el rendimiento académico de estudiantes universitarios" extraídos de la base de datos Scopus en el periodo 2015-2020 distribuidos por autor. Recuperado en 17 de octubre de 2020 de https://www-scopus-com 
Respecto a la afiliación, la mayor cantidad de documentos por afiliación le corresponde a Charles Sturt University, University of Cambridge, Charles Sturt University con 2 documentos para cada caso como se contempla en la figura 3.

\section{Figura 3}

Documentos por afiliación

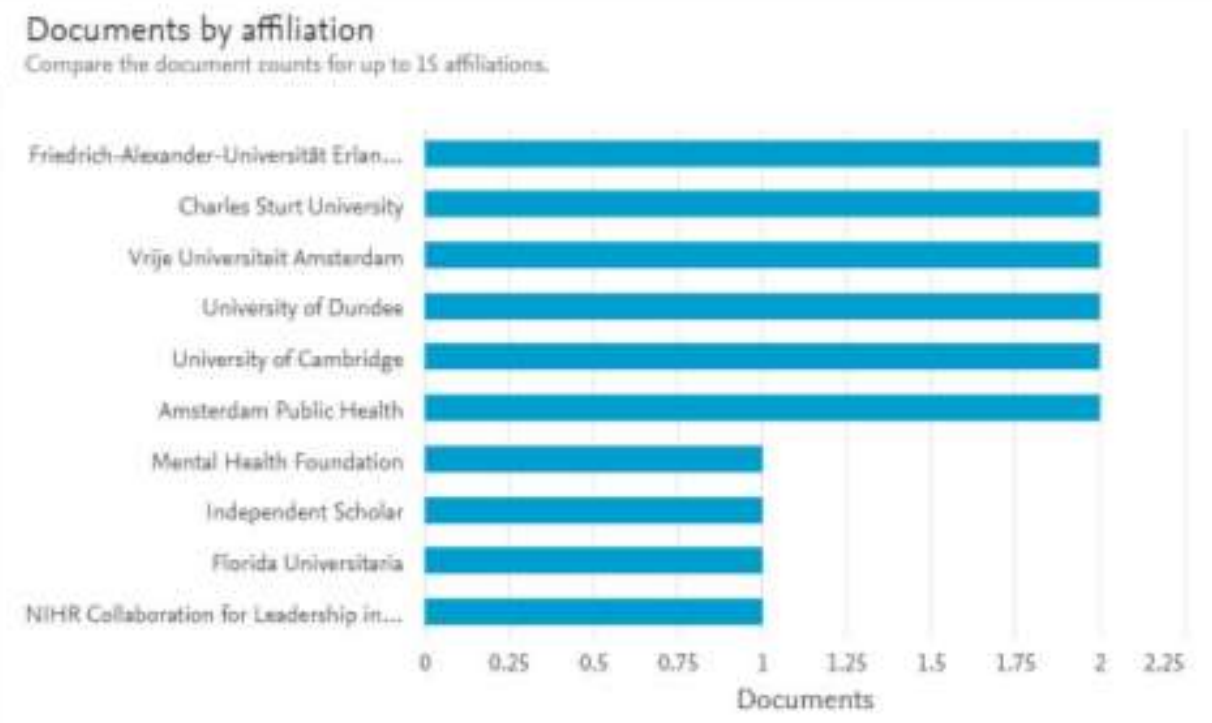

Nota: afiliación institucional de los documentos referidos al tema de investigación "Impacto del mindfulness en el rendimiento académico de estudiantes universitarios" extraídos de la base de datos Scopus en el periodo 20152020. Recuperado en 17 de octubre de 2020 de https://www-scopus-com

A nivel territorial la mayor cantidad de publicaciones se encuentra en Estados Unidos con 18 publicaciones, seguido del Reino Unido con 8 publicaciones, como se puede ver en la figura 4.

\section{Figura 4}

\section{Documentos por ciudad o territorio}

\section{Documents by country or territory \\ Compare the document counts for up to 15 countries/territories.}

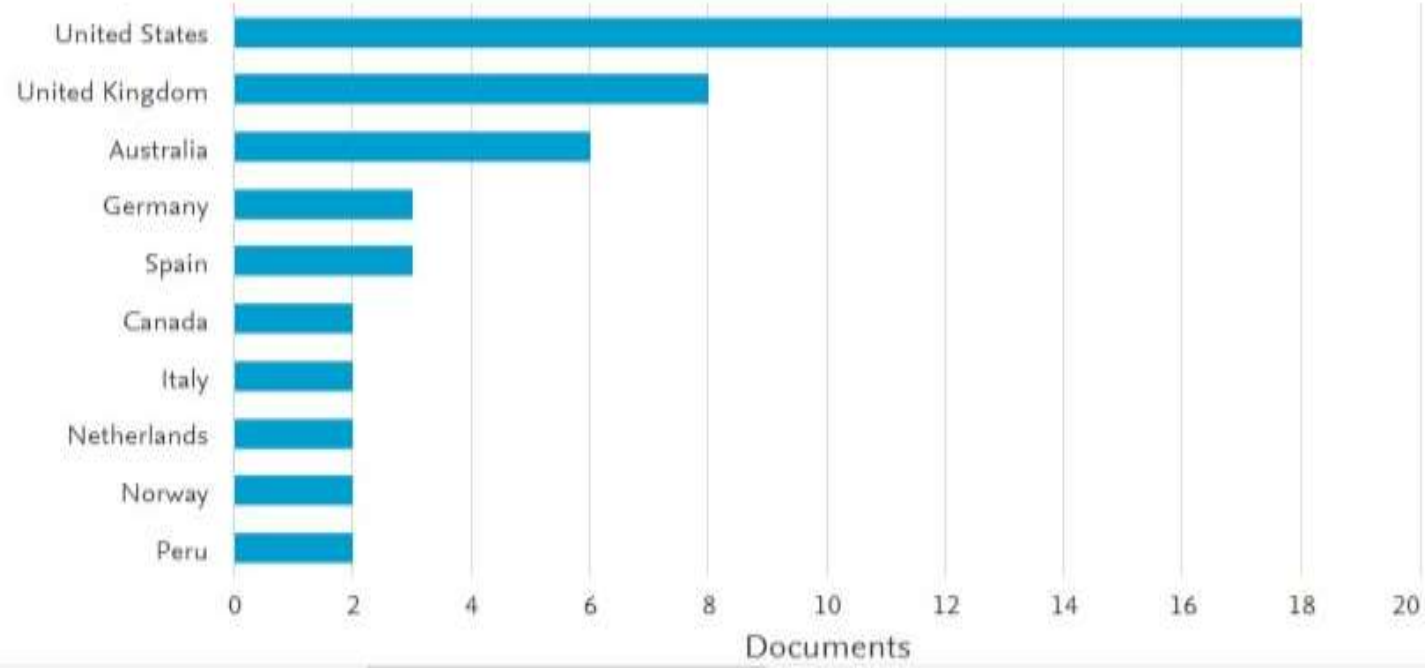

Nota: país de procedencia de los documentos referidos al tema de investigación "Impacto del mindfulness en el rendimiento académico de estudiantes universitarios" extraídos de la base de datos Scopus en el periodo 
2015-2020 distribuidos por ciudad o territorio. Recuperado en 17 de octubre de 2020 de https://www-scopuscom

Respecto a los tipos de documentos la gran mayoría corresponden a artículos $(74,5 \%)$, seguido de Conference paper con 5,9\% como se visualiza en la figura 5 .

\section{Figura 5}

Documentos por tipo

\section{Documents by type}

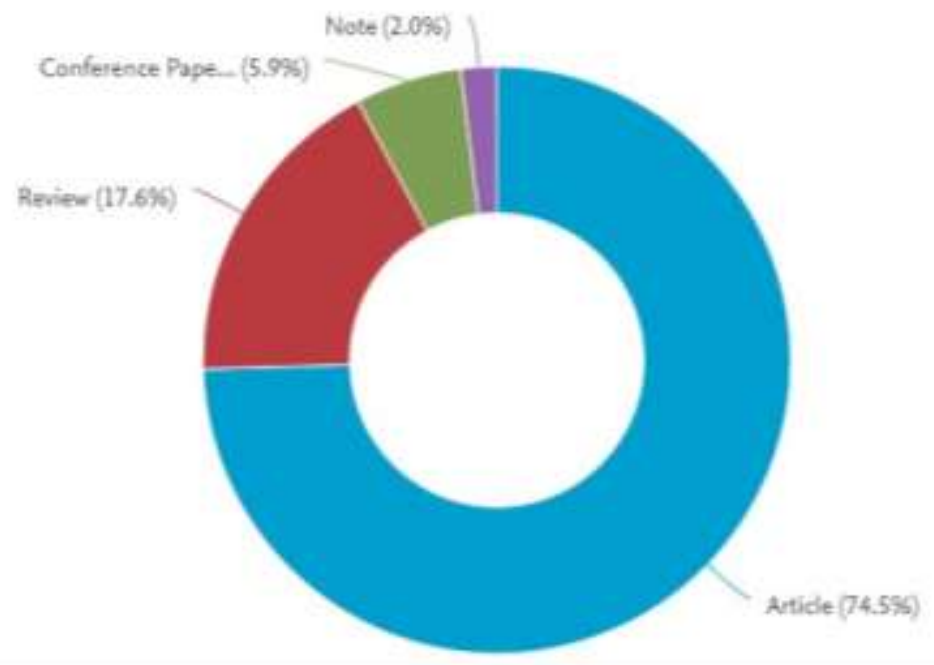

Nota: distribución porcentual según tipo de documentos extraídos de la base de datos Scopus en el periodo 20152020 para el tema de investigación "Impacto del mindfulness en el rendimiento académico de estudiantes universitarios". Recuperado en 17 de octubre de 2020 de https://www-scopus-com

Respecto a la cantidad de documentos por área, se contempla que el tema de investigación se distribuye con un $26,6 \%$ para medicina, $24,1 \%$ para ciencias sociales, $19 \%$ para la psicología, siendo estos los de mayor peso porcentual, como se ve en la figura 6.

\section{Figura 6}

Documentos por área temática

\section{Documents by subject area}

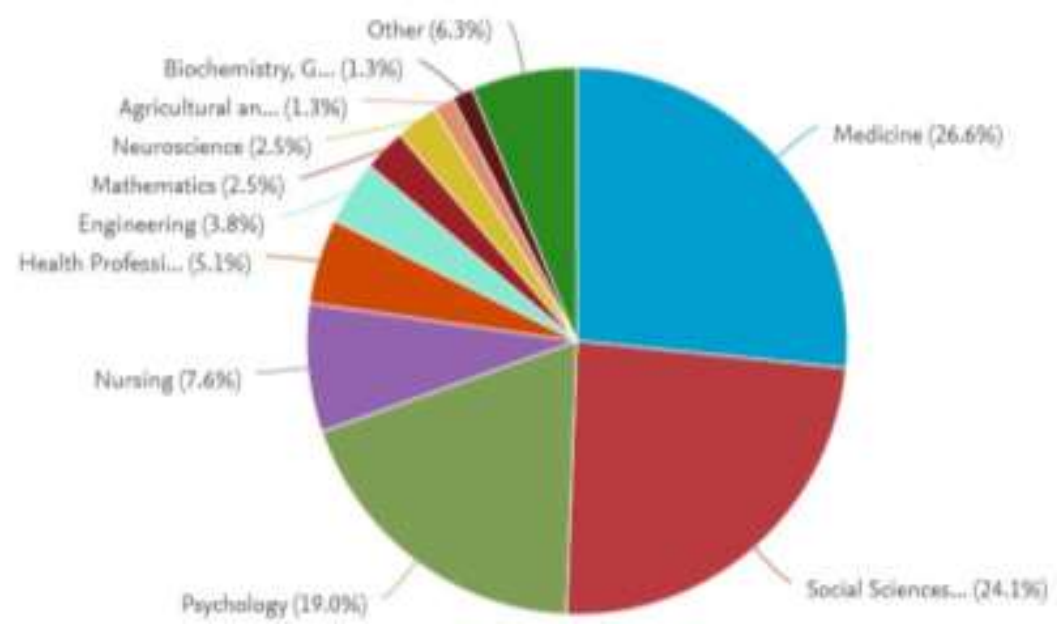


Nota: distribución porcentual por área temática de los documentos extraídos de la base de datos Scopus en el periodo 2015-2020 para el tema de investigación "Impacto del mindfulness en el rendimiento académico de estudiantes universitarios". Recuperado en 17 de octubre de 2020 de https://www-scopus-com

El documento más actual corresponde a "App-based mindfulness meditation for psychological distress and adjustment to college in incoming university students: a pragmatic, randomised, waitlist-controlled trial" de 2020, como se puede ver en la figura 7.

\section{Figura 7}

Documentos más recientes

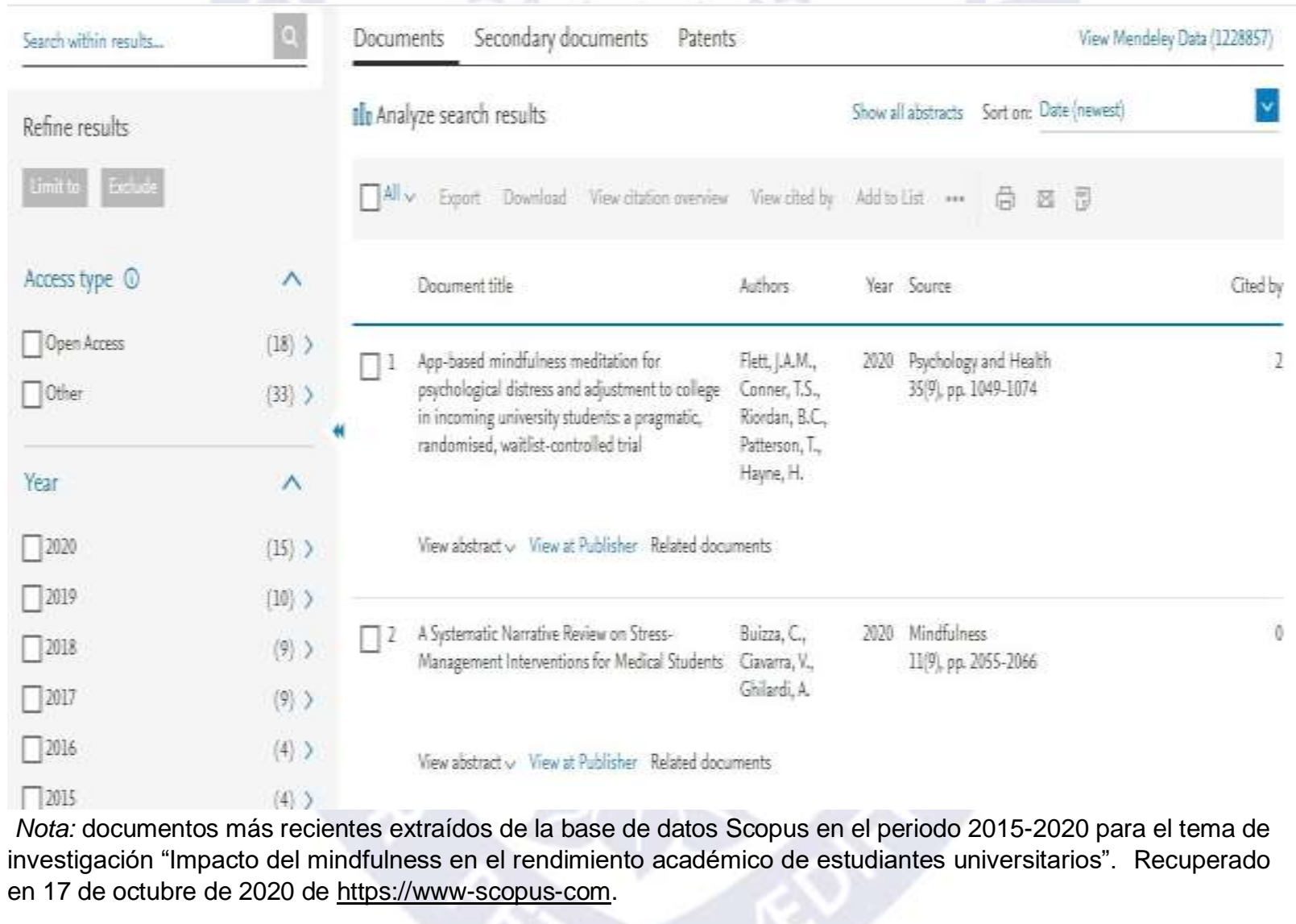

De otro lado, el documento más citado corresponde a McConville, J., McAleer, R., con 59 citaciones como se puede observar en la figura 8. 


\section{Figura 8}

Documentos más citados

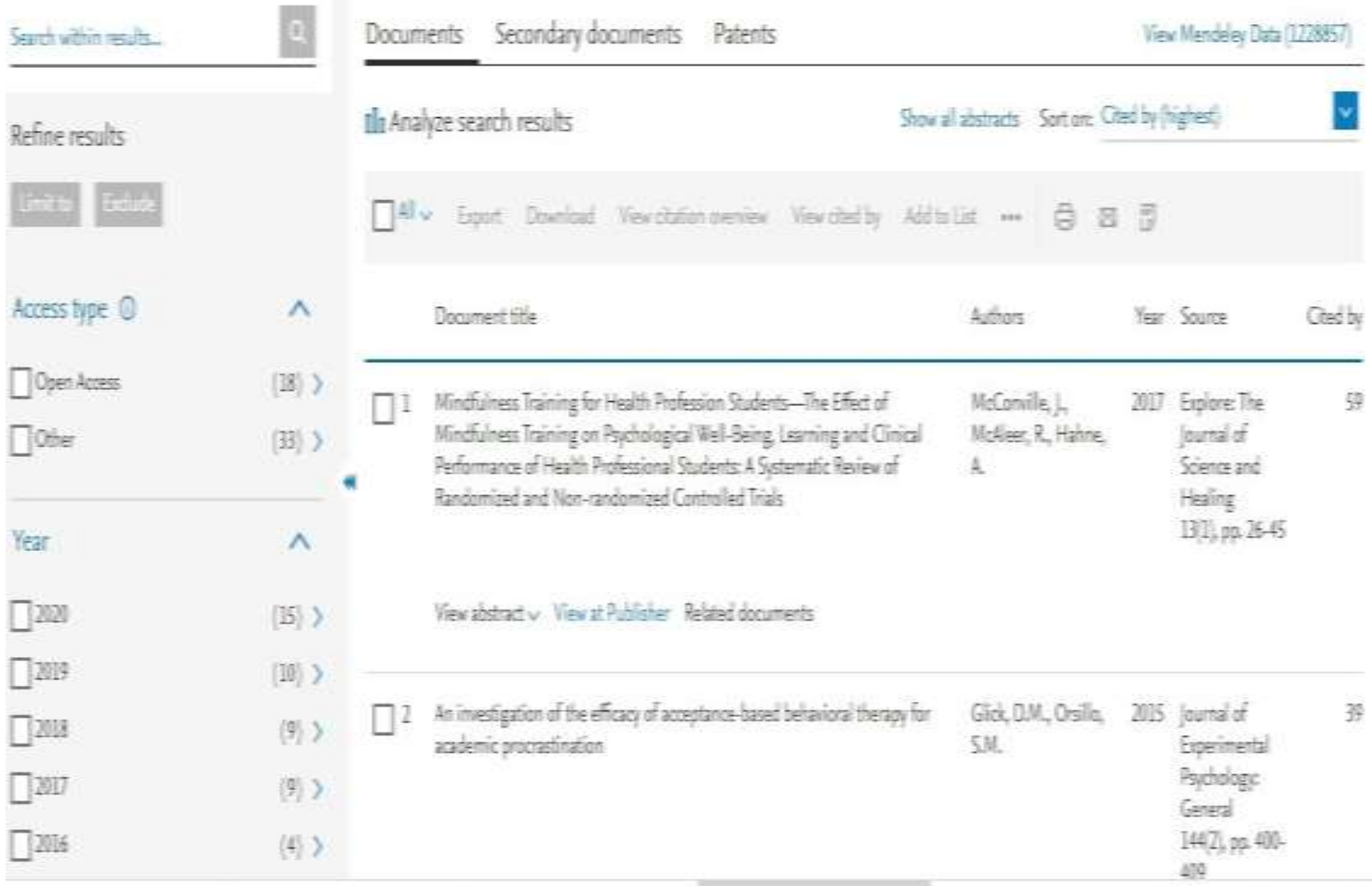

Nota: documentos más citados extraídos de la base de datos Scopus en el periodo 2015-2020 para el tema de investigación "Impacto del mindfulness en el rendimiento académico de estudiantes universitarios". Recuperado en 17 de octubre de 2020 de https://www-scopus-com

El documento con mayor relevancia (el que incorpora la mayor cantidad de términos de la ecuación de búsqueda) es el siguiente "A follow-up study to a randomised control trial to investigate the perceived impact of mindfulness on academic performance in university students". Esto se aprecia en la figura 9. 


\section{Figura 9}

Documento más relevante

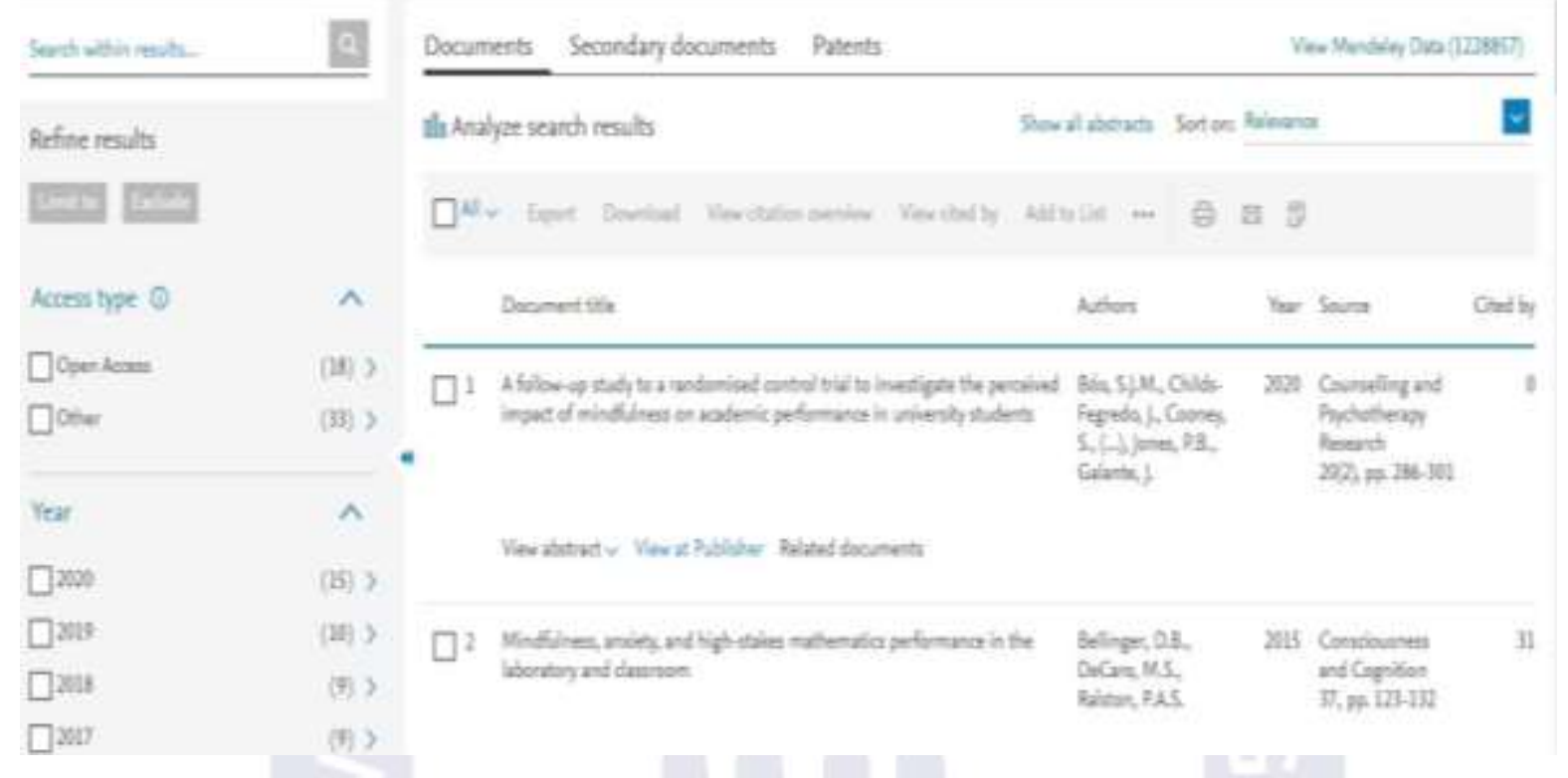

Nota: muestra los primeros 2 documentos que incluyen la mayor cantidad de términos. Recuperado en 17 de octubre de 2020 de https://www-scopus-com.

Para el análisis todos los documentos fueron exportados desde Scopus en formato RIS y luego importados desde Mendeley.

Se ha trabajado únicamente con la base bibliométrica Scopus porque es la que nos permite obtener "el máximo de información posible" (Icts, 2020). WoS integra "base de datos a partir de artículos publicados en las que han sido identificadas como las principales revistas académicas" (Cortés, 2015).

\section{Herramientas de análisis}

\section{Gestor de Referencias}

Se ha usado Mendeley, el que se constituye en un gestor de bibliografía, buscador de información científica, además de ser un sistema que ayuda en el almacenamiento y organización de documentos y se erige además como una red social de tipo académico que permite compartir referencias bibliográficas y documentos de investigación (Rodríguez Otero, 2015). Para el presente trabajo este gestor de referencias fue empleado básicamente para el almacenamiento de documentos. 


\section{Programa de procesamiento de datos}

Se ha empleado VOSviewer que es una herramienta de software para la creación de mapas basados en datos de red (Ritchie et al., 2008) desarrollada en el Centre for Science and Technology Studies (CWTS) (Revista et al., 2016). Se han creado mapas y también se ha realizado visualizaciones de los mismos.

Para el procesamiento se aplicó la ecuación de búsqueda en Scopus y desde allí se exportó por el método CSV Excel a VOSviewer desde donde se hizo la importación para el análisis.

En cuanto a la metodología, VOSviewer utiliza una metodología cuantitativa para la construcción de los mapas bibliométricos, para lo que se requirió de i) recopilación de datos, ii) selección de las unidades de análisis, iii) cálculo de la frecuencia de coocurrencias y coautorías por países y autores y iv) visualización de las correspondientes unidades de análisis en mapas bidimensionales.

\section{Ecuación de la búsqueda}

Para el tema: "Impacto del mindfulness en el rendimiento académico universitario" se usó la ecuación de búsqueda que aparece en la figura 10.

\section{Figura 10}

Ecuación de búsqueda en Scopus

(TITLE-ABS-KEY (mindfulness) AND TITLE-ABS-KEY (academic AND achievement) OR TITLE-

ABS-KEY (academic AND performance) OR TITLE-ABS-

KEY (student AND achievement) OR TITLE-ABS-KEY (student AND progress) AND TITLEABSKEY (higher AND education AND institutions) OR TITLE-ABS-KEY (university) OR TITLEABSKEY (universities) OR TITLE-ABS-KEY (faculty)) AND (LIMIT-

TO (PUBYEAR, 2020) OR LIMIT-TO (PUBYEAR, 2019) OR LIMIT-

TO (PUBYEAR, 2018) OR LIMIT-TO (PUBYEAR, 2017) OR

LIMITTO (PUBYEAR, 2016) OR LIMIT-TO (PUBYEAR, 2015)) 
En la búsqueda se ha empeklado criterios de inclusión y exclusión. Las palabras elegidas para la ecuación han sido aquellas que pudieran permitir mayor precisión en el estudio en función al tema de investigación para así lograr acotar la búsqueda, esto sin dejar de incluir palabras asociados para los términos en el idioma inglés. Para esto se recurrió a Tesauro de la UNESCO, el que se constituye "en una lista controlada y estructurada de términos para el análisis temático y la búsqueda de documentos y publicaciones en los campos de la educación, cultura, ciencias naturales, ciencias sociales y humanas, comunicación e información" (TESAURO de la UNESCO, 2020).

A modo de ejemplo, en la figura 11 se puede notar lo que fue arrojado en TESAURO de la UNESCO sobre "rendimiento académico", uno de los términos de la ecuación de búsqueda en Scopus.

Figura 11

Términos relacionados a rendimiento académico

\begin{tabular}{|c|c|c|}
\hline CONCEPTOS RELACIONADOS & \multicolumn{2}{|l|}{$\begin{array}{l}\text { Control de rendimiento escolar } \\
\text { Eficacia del docente } \\
\text { Evaluación del estudiante } \\
\text { Norma académica } \\
\text { Psicologia de la educación }\end{array}$} \\
\hline PERTENECE AL GRUPO & \multicolumn{2}{|c|}{ Educación > Evaluaciôn de la educaciôn } \\
\hline EN OTRAS LENGUAS & 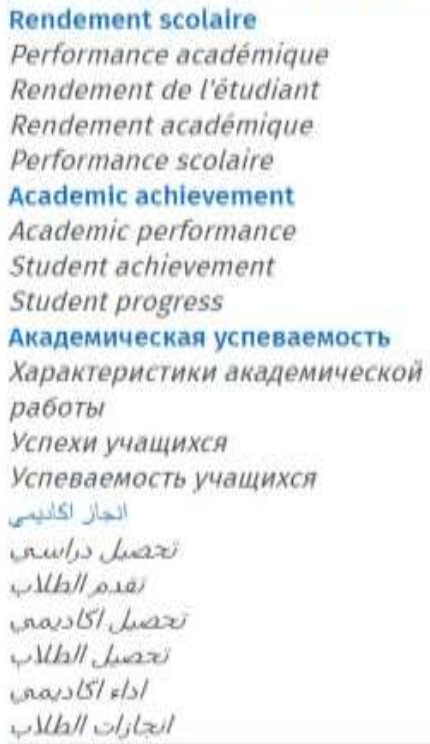 & inglés \\
\hline
\end{tabular}

Nota: términos sinónimos de la palabra "rendimiento académico" en otros idiomas. Recuperado en 17 de octubre de 2020 de http://vocabularies.unesco.org. 
De esta manera se obtuvieron los siguientes términos como sinónimos para la inclusión en la búsqueda:

- Academic achievement, academic performance, student achievement, student progress (rendimiento académico).

- Higher education institutions, university, universities, faculty (Universidad)

\section{Resultados y análisis por coocurrencia de todas las palabras claves con mínimo de ocurrencia de 2 palabras claves}

- Tipo de análisis: coocurrencia

- Unidad de análisis: todas las palabras claves

\section{Análisis de visualización por superposición}

Según el gráfico de la figura 12 se puede observar que el término mindfulness (atención plena) tiene un círculo de gran tamaño, lo que indicaría alta importancia del ítem. Asimismo, las publicaciones con la palabra correlational study (estudio correlacional) al estar más hacia la derecha en la barra de color en la esquina inferior (con color amarillo) indicarían mayor impacto (palabra clave para publicaciones más recientes alrededor del 2020). Lo mismo ocurre para academic performance (desempeño académico), que aparece con el mismo color.

Estos dos últimos términos no se distinguen bien en la figura 12, ya que VOSviewer oculta etiquetas cuando están superpuestas, pero se pueden ver mejor en las figuras 13 y 14 (figuras ampliadas).

Figura 12

Mapa de superposición de palabras claves 
Revista sobre Educación y Sociedad, 2021, 17(1)

DOI: https://doi.org/10.35756/educaumch.202117.151

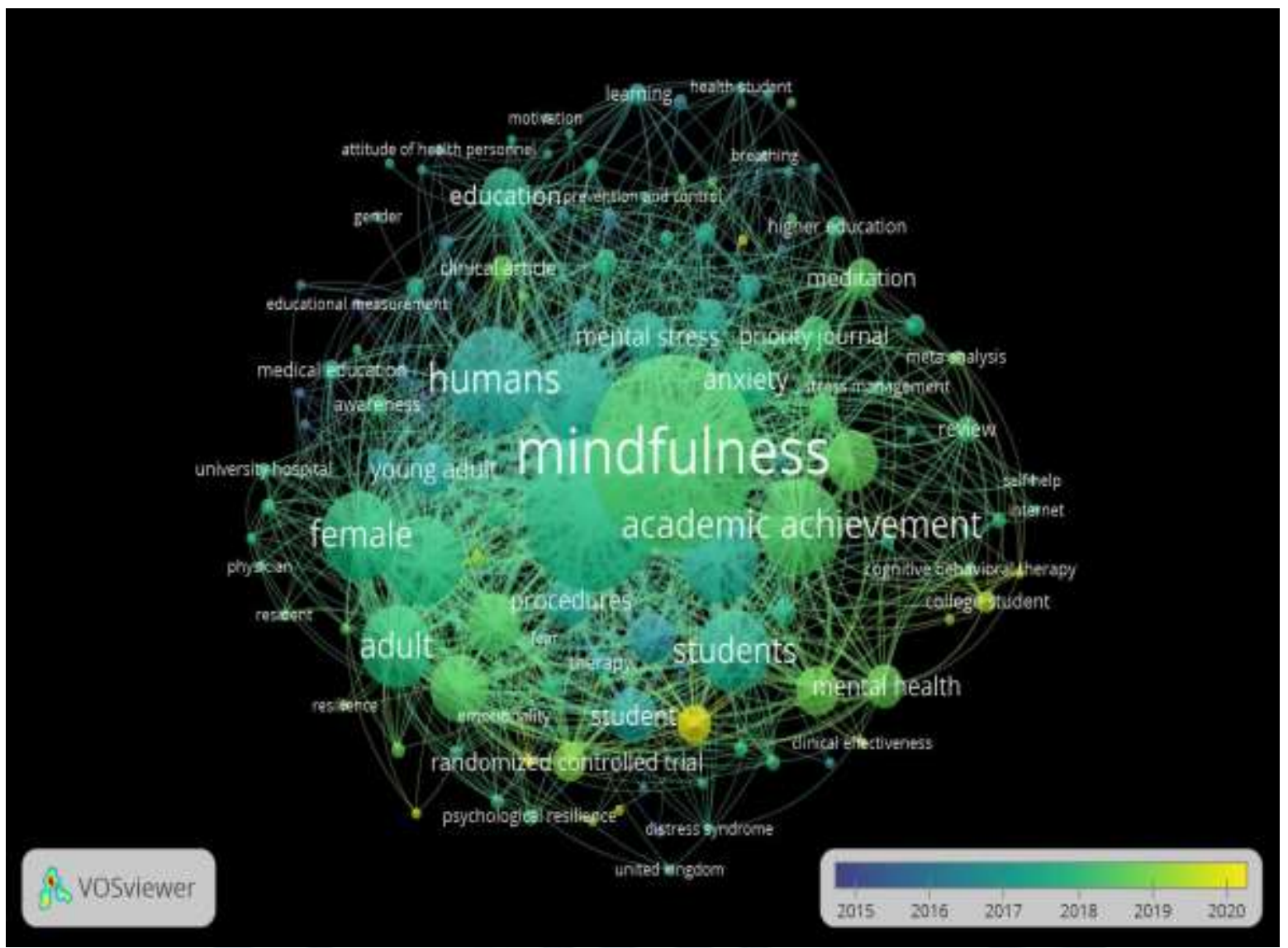

Nota: mapa sobre coocurrencia de todas las palabras claves con mínimo de ocurrencia de 2 palabras. Recuperado en 17 de octubre de 2020 de http://www.vosviewer.com/

Figura 13

Mapa ampliado de superposición de palabras claves 
Revista sobre Educación y Sociedad, 2021, 17(1)

DOI: https://doi.org/10.35756/educaumch.202117.151

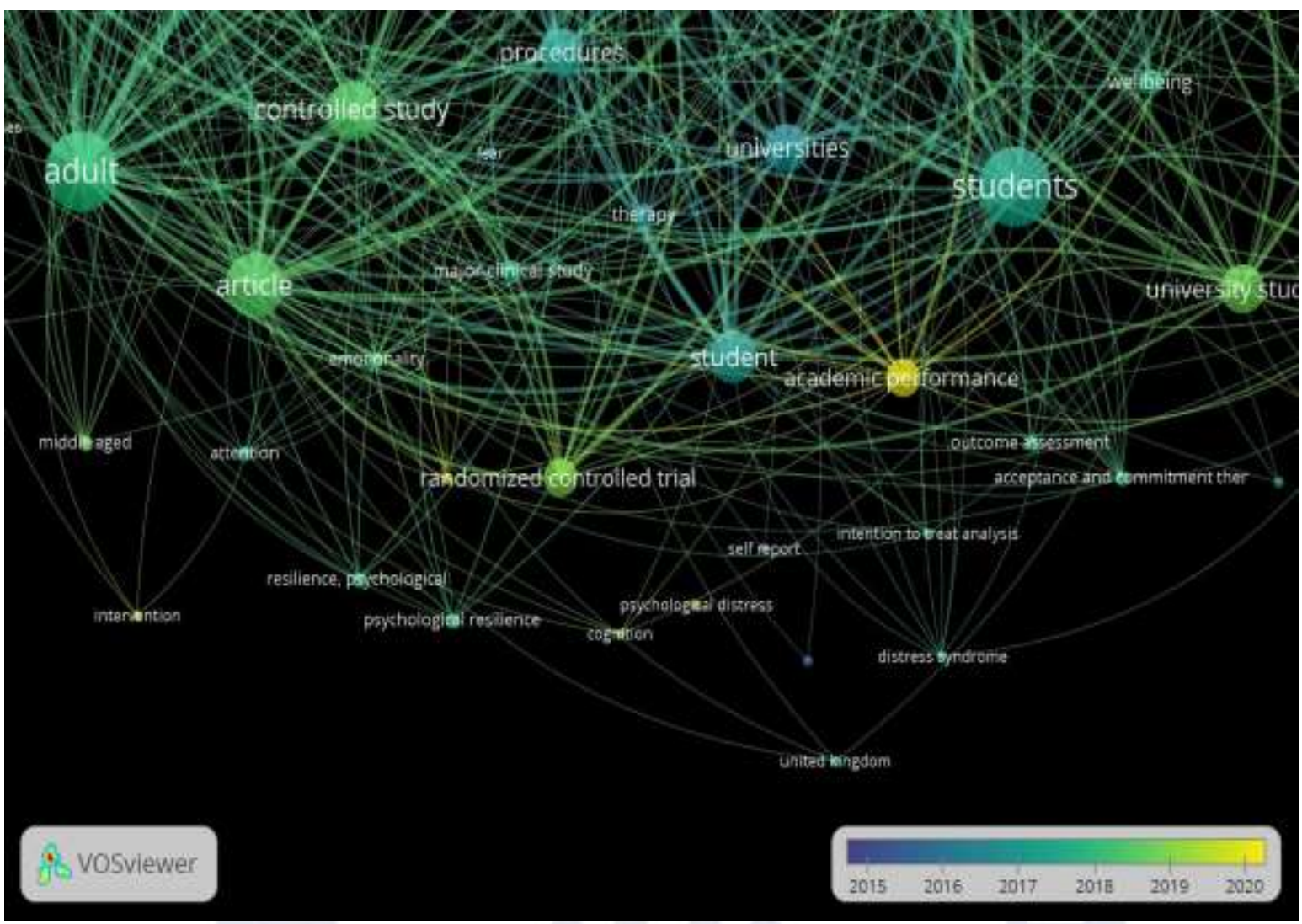

Nota: mapa ampliado sobre coocurrencia de todas las palabras claves con mínimo de ocurrencia de 2 palabras. Recuperado en 17 de octubre de 2020 de http://www.vosviewer.com

\section{Figura 14}

Mapa ampliado de superposición de palabra clave

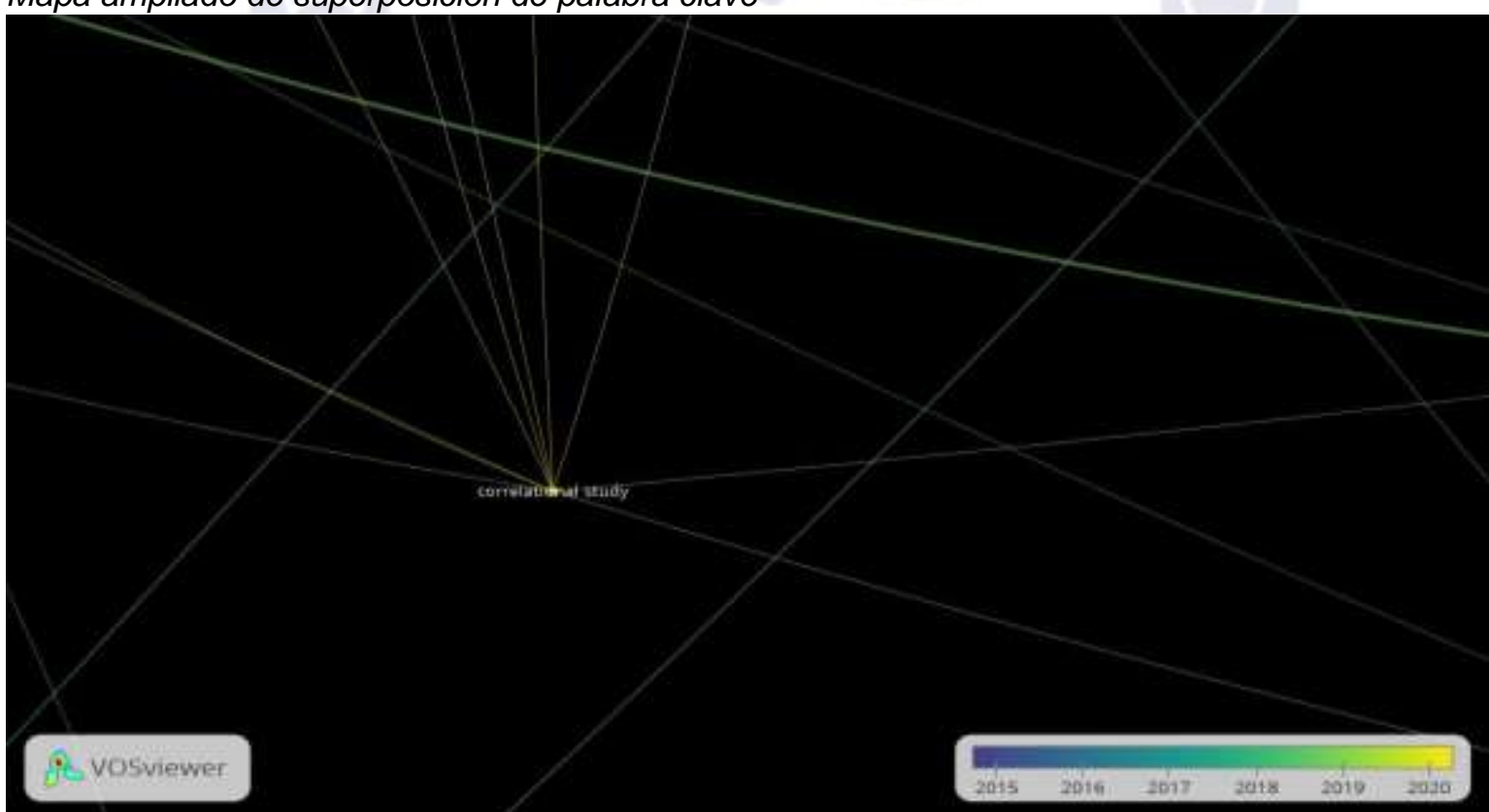

Nota: mapa de superposición sobre coocurrencia de palabra clave con mínimo de ocurrencia de 2 palabras. Recuperado en 17 de octubre de 2020 de http://www.vosviewer.com

Análisis de visualización de red 
Según el análisis se puede notar que la palabra mindfulness aparece formando parte del cluster 1 con términos como academic performance (desempeño académico), cognition (cognición), emotionality (emocionalidad), entre otros. El cluster 2 se constituye así en una red de 33 ítems, mientras que el cluster 1 lo componen 38 ítems, el 3 lo conforman 29 ítems y el cluster 4 lo constituyen 25 ítems. Se presentan un total de 4 clusters.

\section{Análisis de visualización por densidad}

De otro lado, haciendo el análisis de densidad se puede observar que las palabras en colores más intensos son a los que les corresponden mayor número de ocurrencias. Así se puede ver en la figura ampliada 15 que las palabras mindfulness y human (35 y 28 ocurrencias respectivamente) tienen un nodo de color más intenso que el de palabras como perception (percepción) y mental performance (desempeño mental) con 4 y 3 ocurrencias respectivamente.

\section{Figura 15}

Mapa de densidad de palabras claves

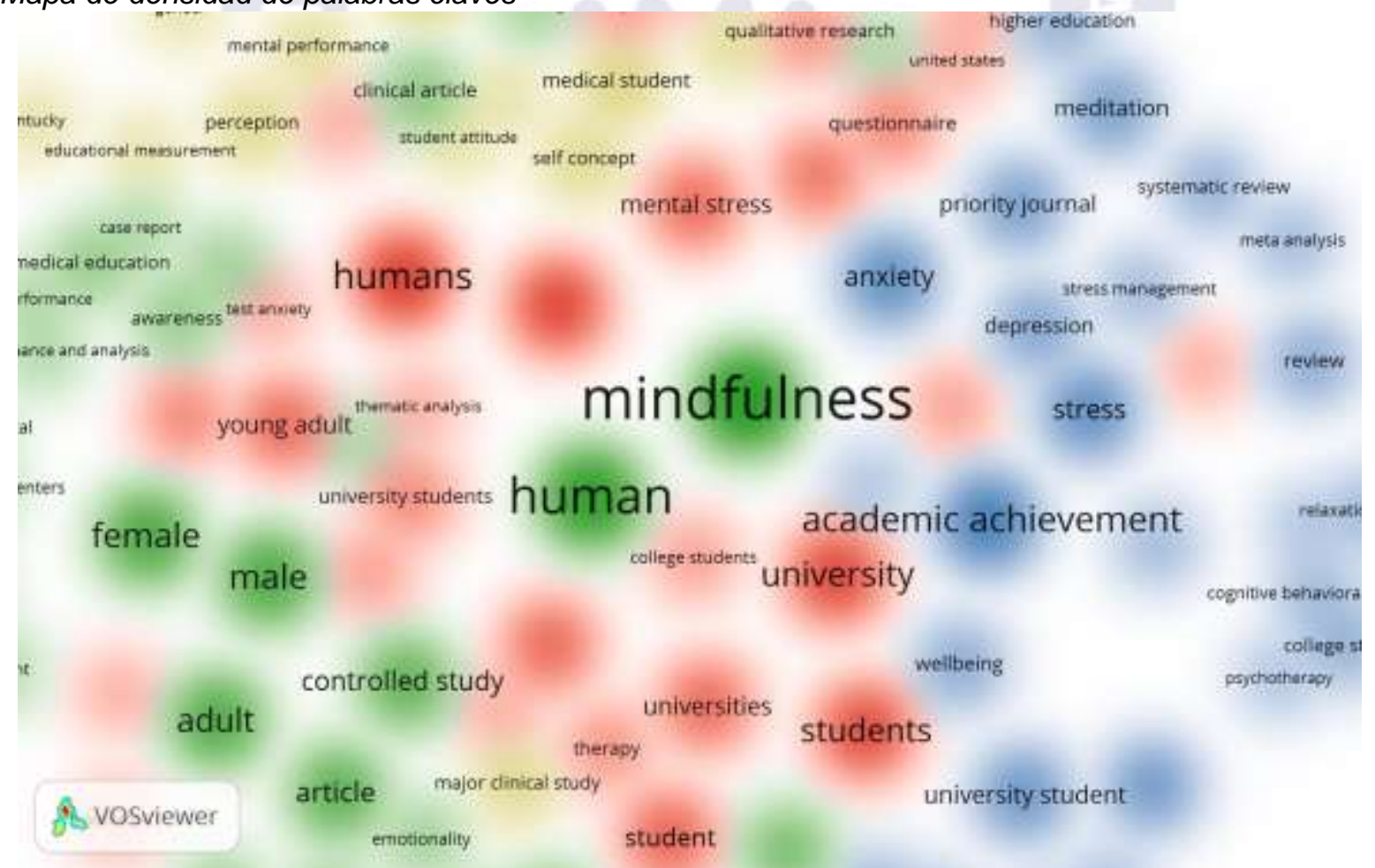

Nota: mapa sobre coocurrencia de todas las palabras claves con mínimo de ocurrencia de 2 palabras. Recuperado en 17 de octubre de 2020 de http://www.vosviewer.com

Sin embargo, se contempla que la repetición de palabras como "human", "humans", "article", “case report" no dan mucha significancia, por lo que se volvió a hacer el análisis excluyéndolas. 
Se pensó inicialmente en excluir también del análisis a "male" y "female", sin embargo, sería interesante saber sobre una probable asociación entre el mindfulness y el género, pero al observar la tabla de ocurrencias tanto hombres como mujeres aparecían con la misma cantidad de ocurrencias (16), por lo que no se constituiría el género en variable de relevancia. En consecuencia, estos dos términos también fueron excluidos. Se obtiene así, una nueva visualización de red (ver figura 16).

\section{Figura 16}

Mapa de superposición excluyendo términos

\section{vosviewer}

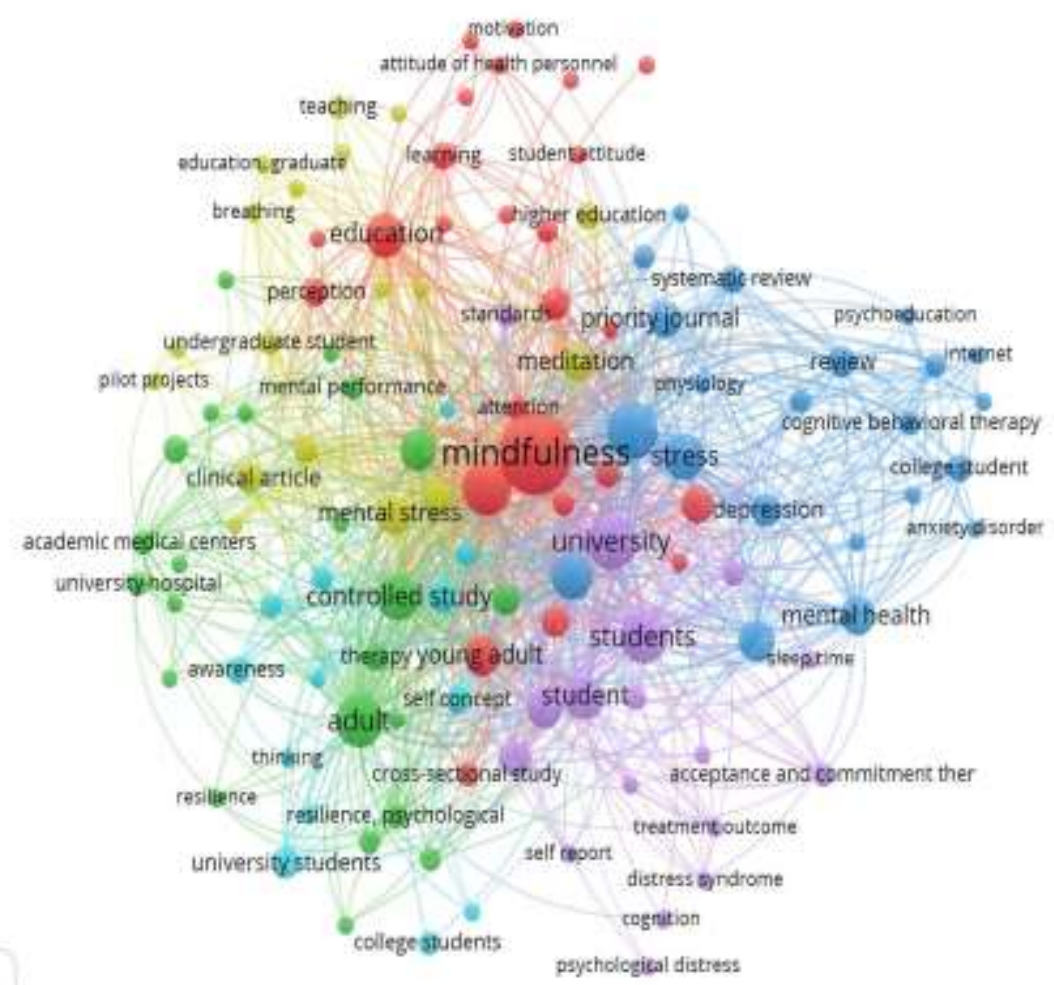

Nota: mapa sobre coocurrencia de todas las palabras claves con mínimo de ocurrencia de 2 palabras, excluyendo "human", "humans", "article" y "case report. Recuperado en 17 de octubre de 2020 de http://www.vosviewer.com

Se distingue que las palabras mindfulness (atención plena) tiene la mayor cantidad de ocurrencias (36), seguidamente de las siguientes: academic achievement o logro académico (17), university o universidad (16) y psychology o psicología (16). En la figura 17 se aprecia que la palabra mindfulness tiene una gran cantidad de enlaces (links) con muchos otros términos claves.

\section{Figura 17}

Mapa de superposición ampliado de palabras claves con exclusión de términos 
Revista sobre Educación y Sociedad, 2021, 17(1)

DOI: https://doi.org/10.35756/educaumch.202117.151

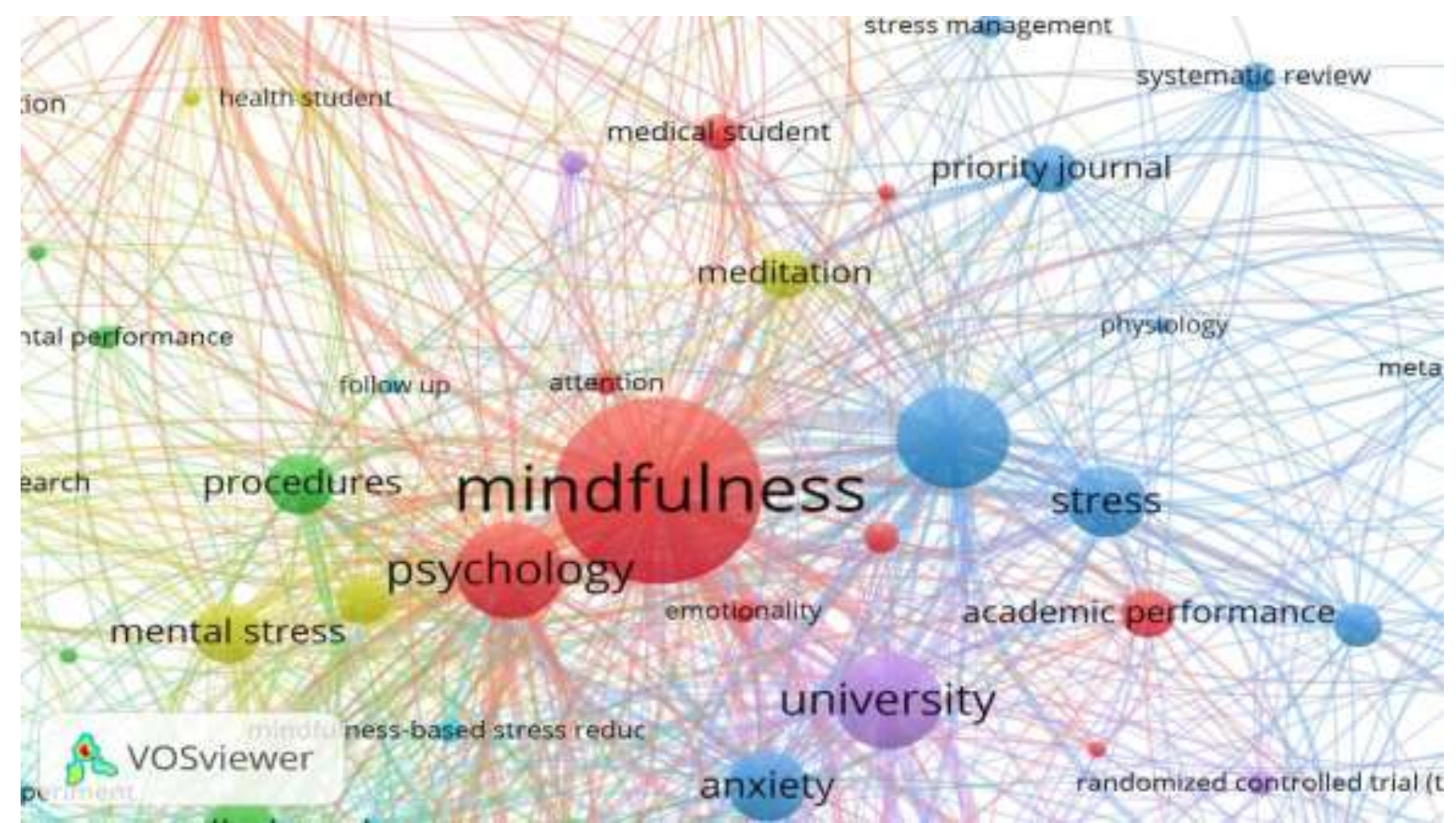

Nota: mapa sobre coocurrencia de todas las palabras claves con mínimo de ocurrencia de 2 palabras, excluyendo "human", "humans", "article" y "case report. Recuperado en 17 de octubre de 2020 de http://www.vosviewer.com

\section{Análisis por coautoría por países}

- Tipo de análisis: coautoría

- Unidad de análisis: países

\section{Análisis de ratio citaciones/documentos por ciudad}

Se realizó un análisis de citaciones considerando 1 como mínimo número de citaciones de un país y 3 como mínimo número de documentos según se aprecia en la tabla 1 .

\section{Tabla 1}

Ratio citaciones/documentos por ciudad con mínimo de 1 citación por país y 3 documentos como mínimo

\begin{tabular}{lccc}
\hline Ciudad & Documentos & Citaciones & $\begin{array}{c}\text { Ratio } \\
\text { citaciones } / \text { documentos }\end{array}$ \\
\hline Estados Unidos & 17 & 117 & $=117 / 17=16,71$ \\
Australia & 6 & 66 & $=66 / 6=11$ \\
Alemania & 3 & 46 & $=46 / 3=15,33$ \\
Reino Unido & 8 & 40 & $=40 / 8=5$ \\
España & 3 & 7 & $=7 / 3=2,33$ \\
\hline
\end{tabular}

Fuente: elaboración propia

Nota: la tabla muestra los países con mayor citación promedio por documento 
De la tabla se puede notar una mayor producción de documentos en Estados Unidos y la más baja producción en España, con 117 citaciones y 7 citaciones respectivamente. De otro lado, aparentemente Australia tiene mejor ubicación que Alemania si se ve únicamente la columna de citaciones, pero si se mira los ratios calculados en la última columna, se distingue que el ratio es de 15,33 en Alemania versus 11 en Australia.

\section{Análisis de visualización de red}

Se puede apreciar la generación de 1 cluster entre Alemania y Reino Unido, mientras que Estados Unidos pertenece a otro cluster (con otro color).

Asimismo, en cuanto a documentos se ve que Estados Unidos tiene una mayor cantidad que Reino Unido y a su vez Reino Unido respecto a Alemania (detectado por el tamaño del rectángulo), tal como se observa en la figura 18 y como se apreció en la tabla 1.

\section{Figura 18}

Mapa de visualización de red por coautoría por países

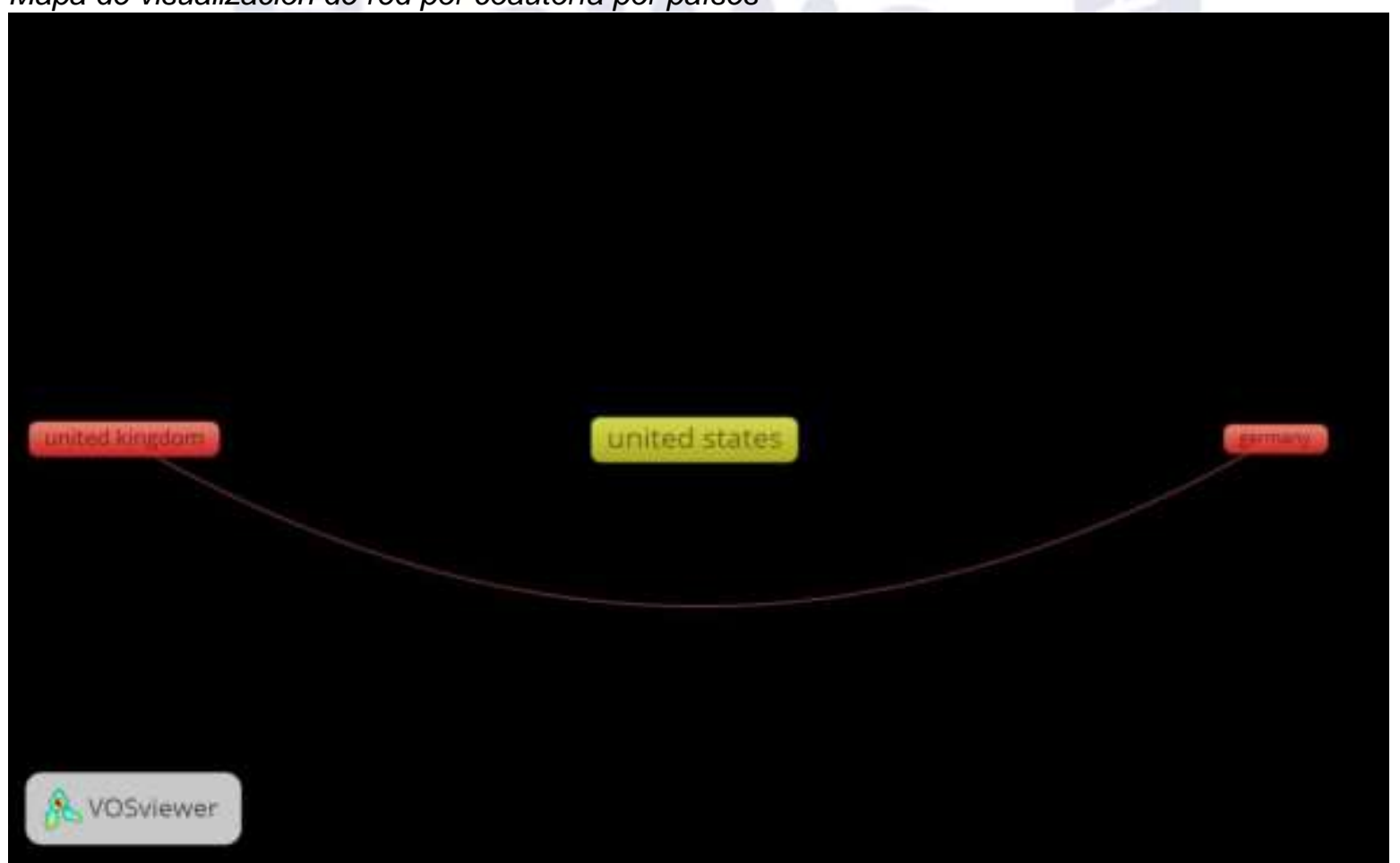

Nota: se contempla 1 como mínimo número de citaciones de un país y 3 como mínimo número de documentos. Recuperado en 17 de octubre de 2020 de http://www.vosviewer.com

\section{Análisis de visualización por densidad}


Lo mencionado en el punto anterior se puede corroborar con la figura de densidad o de calor (ver figura 19); donde en orden descendente, la densidad en los siguientes países es como sigue: Estados Unidos, Reino Unido y Alemania.

\section{Figura 19}

Mapa de densidad por coautoría por países

Nota: contempla 1 como mínimo número de citaciones de un país y 3 como mínimo número de documentos. Recuperado en 17 de octubre de 2020 de http://www.vosviewer.com

\section{Análisis de visualización por superposición}

Si lo que se quiere saber es qué país es el que tiene las publicaciones con más impacto este sería Alemania (cuyo color de rectángulo se acerca al extremo derecho en la barra inferior) en comparación con Estados Unidos y Reino Unido. Ver figura 20. 


\section{Figura 20}

\section{Mapa de visualización por superposición por coautoría por países}

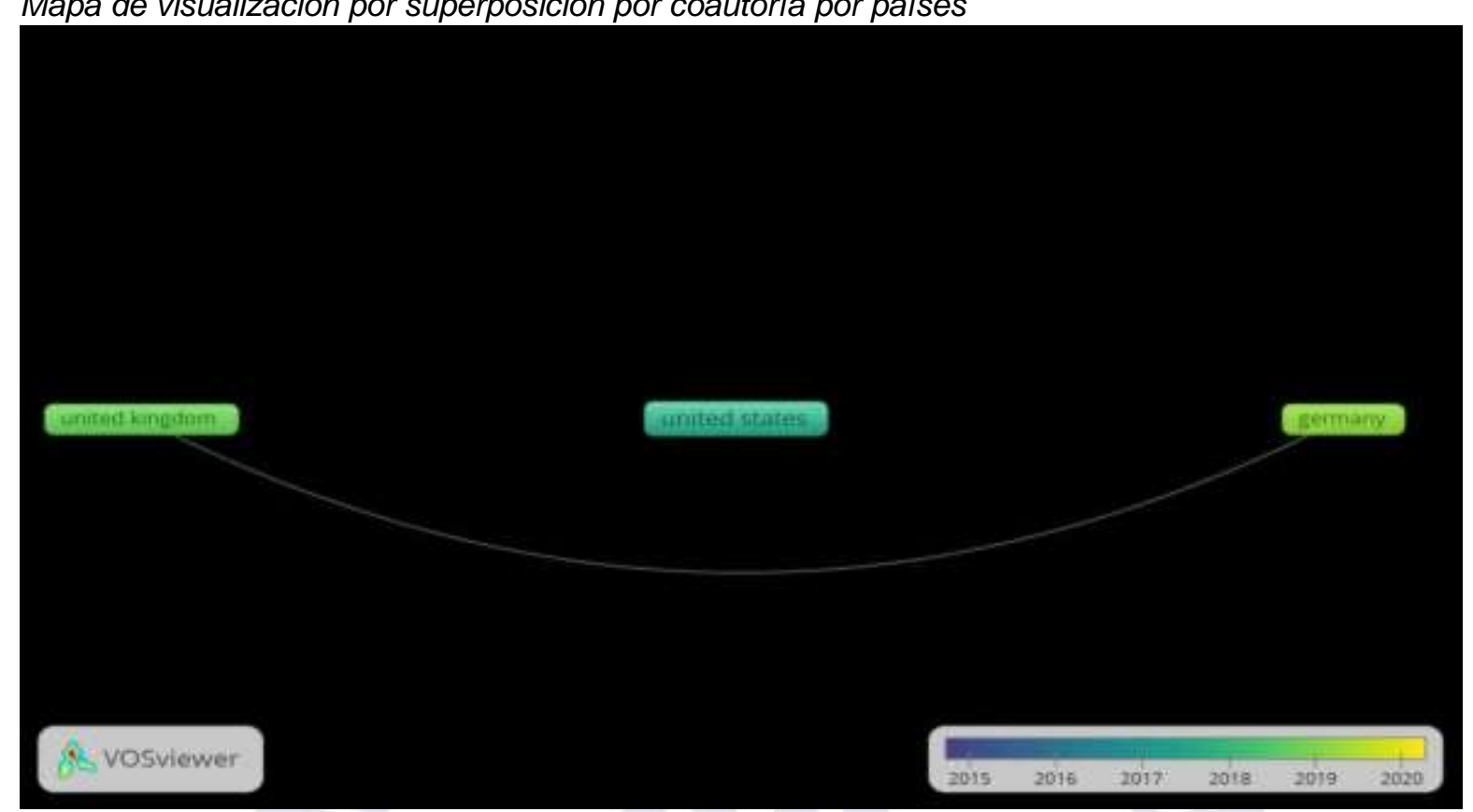

Nota: contempla 1 como mínimo número de citaciones de un país y 3 como mínimo número de documentos. Recuperado en 17 de octubre de 2020 de http://www.vosviewer.com

\section{Análisis de coautoría en autores}

\section{Análisis de ratio citaciones/documentos por autor primer escenario}

Se hizo un análisis considerando un mínimo de 2 documentos por autor y un mínimo de 1 citación de autor como se aprecia en la tabla 2.

\section{Tabla 2}

Ratio citaciones/documentos por autor con mínimo de 2 documentos por autor y 1 citación de autor

\begin{tabular}{lcccc}
\hline Autor & $\begin{array}{l}\text { Document } \\
\text { os }\end{array}$ & Citaciones & $\begin{array}{c}\text { Fortaleza total del } \\
\text { link }\end{array}$ & $\begin{array}{c}\text { Ratio } \\
\text { citaciones/documentos }\end{array}$ \\
\hline Dufour G. & 2 & 15 & 4 & $=15 / 2=7,5$ \\
Galante J. & 2 & 15 & 4 & $=15 / 2=7,5$ \\
Jones P. B. & 2 & 15 & 4 & $=15 / 2=7,5$ \\
Cuijpers P. & 2 & 11 & 2 & $=11 / 2=5,5$ \\
Ebert D. D. & 2 & 11 & 2 & $=11 / 2=5,5$ \\
Chen J. & 2 & 10 & 0 & $=10 / 2=5$ \\
\hline
\end{tabular}

Fuente: elaboración propia

Nota: la tabla muestra los autores con mayor citación promedio por documento

En la tabla se observa que a Dufour le corresponde la mayor cantidad de citaciones/documentos, lo mismo que Galante y Jones. También distingue a través de la fortaleza total del link que hay una mayor relación respecto a la coautoría en los tres primeros autores de la tabla que con los dos siguientes (4 versus 2). 


\section{Análisis de visualización por superposición primer escenario}

De otro lado, como se puede ver en la figura 21, si se hace la visualización por superposición se originan 3 clusters donde en dos de ellos existen interacciones. Así, los autores Dufour, Jones y Galante comparten enlaces, mientras que Ebert y Cruijpers lo hacen por otro lado. Asimismo, el autor Chen no tiene interacciones, aunque es probable que tenga enlaces con otros autores.

También, se distingue que las interacciones entre Ebert y Cruijpers en la línea de tiempo están más cerca de los últimos años.

\section{Figura 21}

Mapa de visualización por superposición por coautoría en autores según primer escenario
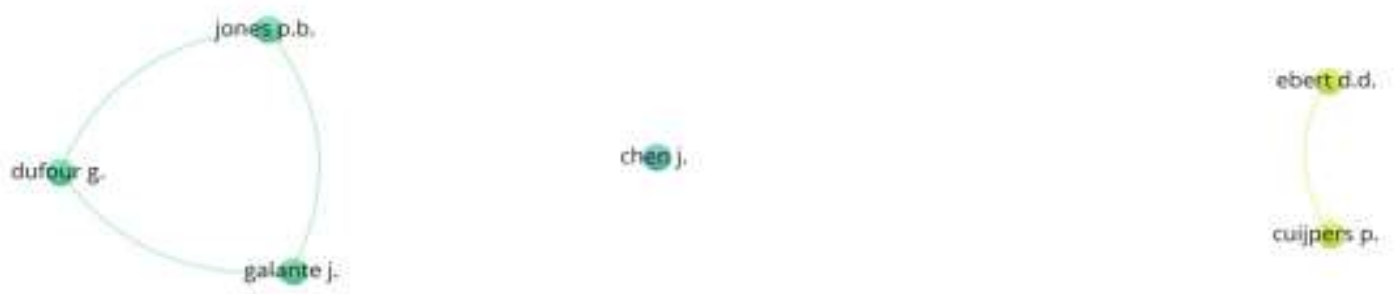

\section{vosviewer}

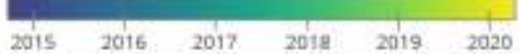

Nota: el primer escenario contempla un mínimo de 2 documentos por autor y un mínimo de 1 citación de autor. Recuperado en 17 de octubre de 2020 de http://www.vosviewer.com

\section{Análisis de ratio citaciones/documentos segundo escenario}

Del análisis se observa que al hacer la búsqueda con mínimo 2 documentos y 1 citación podría haber autores con documentos citados más veces así hayan sido los únicos que han escrito. Es por ello que se determinó también como uno de los objetivos de estudio hacer una búsqueda con un mínimo de 1 documento y 5 citaciones como mínimo, lo que se constituyó en el segundo escenario, con lo cual se obtienen los resultados observados en la tabla 3 donde se han considerado únicamente los primeros 6 registros para la elaboración de la misma. 
Tabla 3

Ratio citaciones/documentos por autor con mínimo de 1 documento por autor y 5 citaciones de autor

\begin{tabular}{lcccc}
\hline Autor & Documentos & Citaciones & $\begin{array}{c}\text { Fortaleza } \\
\text { total del link }\end{array}$ & $\begin{array}{c}\text { Ratio } \\
\text { citaciones/documentos }\end{array}$ \\
\hline Hahne A. & 1 & 58 & 2 & $=58 / 1=58$ \\
Mcaleer R. & 1 & 58 & 2 & $=58 / 1=58$ \\
Mcconville J. & 1 & 58 & 2 & $=58 / 1=58$ \\
Glick D. M. & 1 & 39 & 1 & $=39 / 1=39$ \\
Orsillo S.M. & 1 & 39 & 1 & $=39 / 1=39$ \\
Friedrich A. & 1 & 35 & 1 & $=35 / 1=35$ \\
\hline
\end{tabular}

Fuente: elaboración propia

Nota: la tabla muestra los autores con mayor citación promedio por documento

De la tabla 3 se puede apreciar que la gran mayoría de citaciones/documentos corresponden a documentos únicos de autores como Hahne, Mccaleer y Mcconville con 58 citaciones para todos ellos, seguidos de Glick y Orsillo y prosiguiendo con Friedrich.

Se visualiza también que la fuerza total del enlace entre los 3 primeros autores es distinta que para los 3 últimos, ya que les corresponden numeraciones diferentes en la tercera columna.

Comparando los resultados de la tabla 3 con la tabla 2 se determina que el ratio citaciones/documentos de los autores Hahne, Mccaleer y Mcconville es 7,73 veces el número obtenido con los autores Dufour, Galante y Jones $(58 / 7,5)$ y 10,54 veces el número obtenido por Cuijpers y Ebert $(58 / 5,5)$.

Se complementa la información de la tabla 3 con la figura 22 bajo el criterio de búsqueda de mínimo 1 documento y mínimo 5 citaciones por cada uno:

\section{Análisis de visualización de red (segundo escenario)}

Según la figura 22 se nota el grado de relación entre los autores por medio del color del nodo de las etiquetas. Así, los autores que más referencias tienen (Hahne, Mcconville, Mcaleer) forman parte del mismo cluster. 


\section{Figura 22}

Mapa de visualización por superposición por coautoría en autores según segundo escenario

vosviewer

$$
\begin{aligned}
& \text { rogers } \mathrm{m} \text {. } \\
& \text { gilmartin h. quinh m. } \\
& \text { chopra } \mathrm{v} \text {. } \\
& \text { winters. } \\
& \text { saint } 5 \text {. }
\end{aligned}
$$

Nota: el segundo escenario contempla un mínimo de 1 documento por autor y mínimo de 5 citaciones. Recuperado en 17 de octubre de 2020 de http://www.vosviewer.com

\section{Análisis de visualización por densidad (segundo escenario)}

De otro lado, según el mapa de calor o densidad de la figura 23 y haciendo análisis por citaciones se determina la mayor intensidad para los autores con mayores citaciones.

\section{Figura 23}

Mapa de densidad por coautoría en autores según segundo escenario 


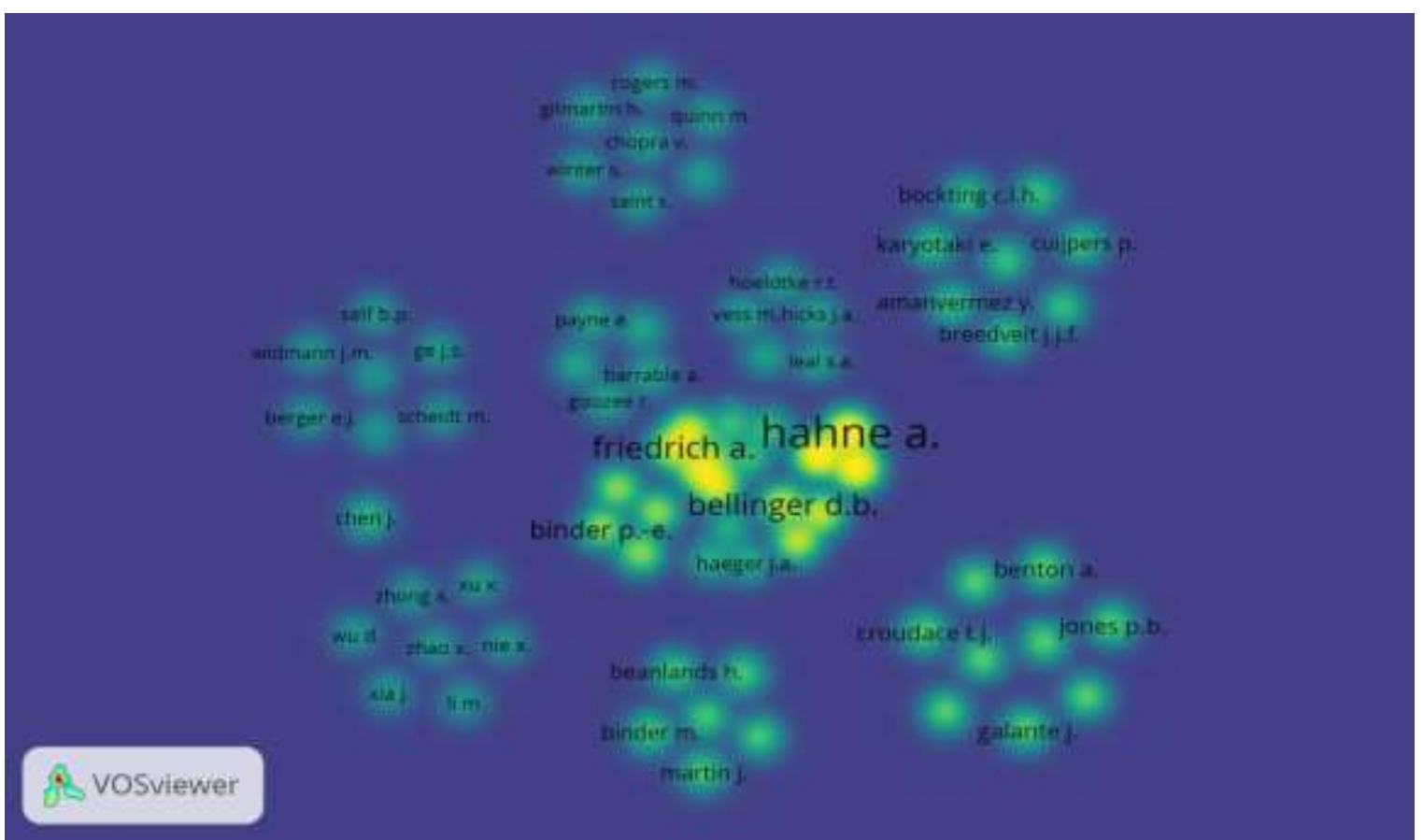

Nota: el segundo escenario contempla un mínimo de 1 documento por autor y mínimo de 5 citaciones. Recuperado en 17 de octubre de 2020 de http://www.vosviewer.com

\section{Análisis de visualización por superposición segundo escenario}

Ante la pregunta ¿acaso los autores más citados lo son porque sus publicaciones son más antiguas o porque son más relevantes?, se ha realizado el análisis con la barra de color. De esta manera, según se aprecia en la figura 24 los autores del cluster amarillo tienen mayor impacto debido a que sus publicaciones son más recientes, como las del 2019 (ver la barra de color inferior derecha). Los autores con publicaciones más recientes corresponden a Amanvermez, Bockting, Breedvelt, Karyotakie y Cuijpers.

De otro lado, a partir de la tabla 3, la cual muestra solo los 6 resultados más altos de citaciones, se sabe que la cantidad de citaciones obtenidas para estos autores fue de 11 para cada uno. Asimismo, la cantidad de citaciones de Hahne, Mccaleer y Mcconville es 5,27 veces la obtenida por Amanvermez, Bockting, Breedvelt, Karyotakie y Cuijpers (58/11). 


\section{Figura 24}

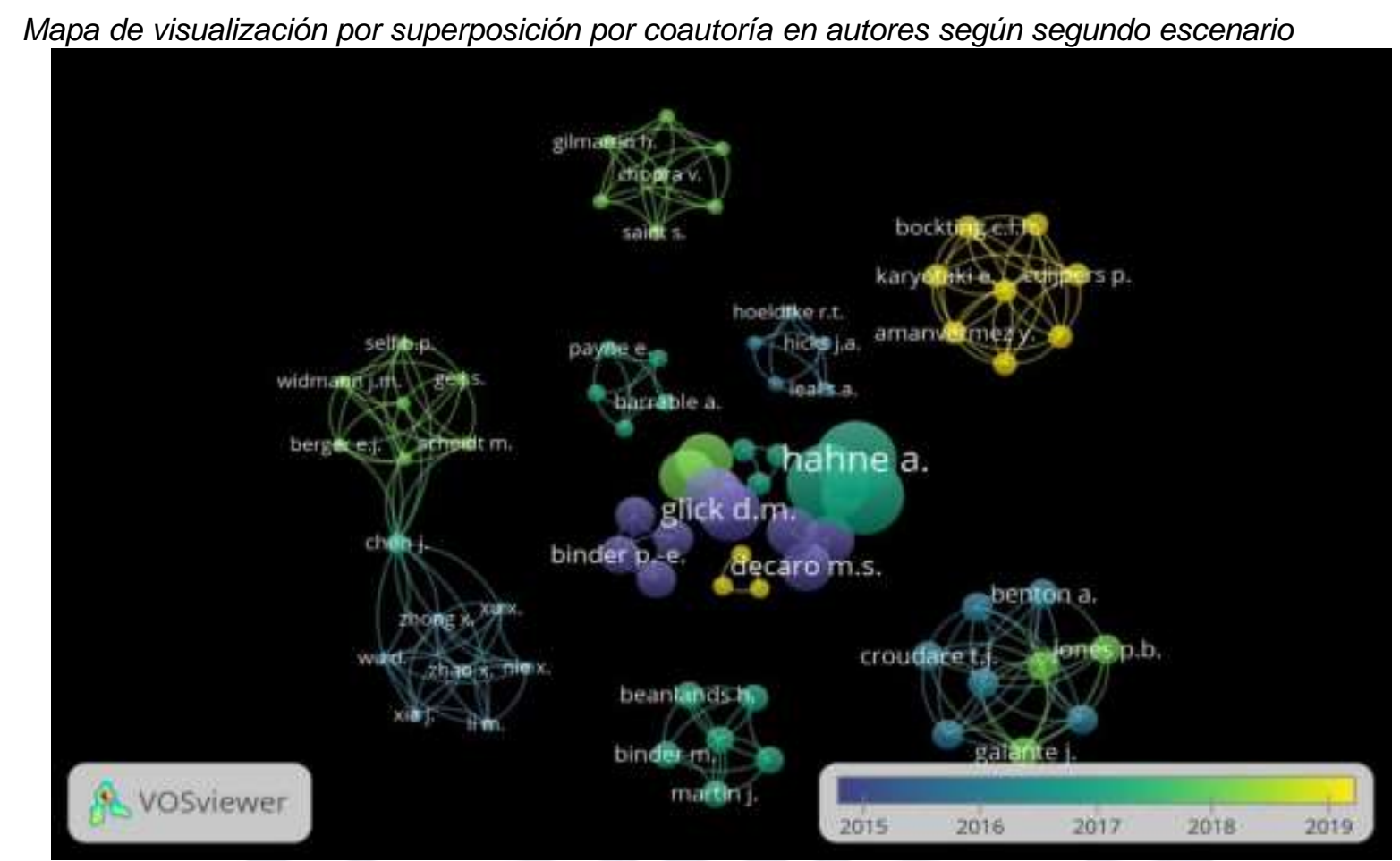

Nota: el segundo escenario contempla un mínimo de 1 documento por autor y mínimo de 5 citaciones. Recuperado en 17 de octubre de 2020 de http://www.vosviewer.com

\section{Conclusiones y recomendaciones}

\section{- Respecto al objetivo general 1}

Las palabras con mayor coocurrencia en todas las palabras claves considerando un mínimo de 2 palabras clave fueron mindfulness y human. Las palabras con mayor ocurrencia al excluir términos no significativos fueron mindfulness, academic achievement, university y psichology, por lo que se concluye que sí ha habido impacto con la exclusión.

Se recomienda ahondar en publicaciones de revistas científicas relacionadas con la psicología por cuanto la atención plena (mindfulness) es un proceso cognitivo y los procesos cognitivos son de interés de la psicología, apreciándose así relación entre mindfulness y palabras como breathing (respiración), anxiety (ansiedad), stress (estrés), resilience (resiliencia), anxiety (ansiedad) y sleep time (tiempo de sueño).

\section{- Respecto al objetivo general 2}

Los países con mayor ratio citación/documentos en orden descendente fueron Estados Unidos, Alemania y Australia. 
Se recomienda consultar documentos referidos al tema de investigación y que hayan sido producidos en los países antes mencionados, aunque con publicaciones de Alemania o Reino Unido sería poco probable llegar a publicaciones de Estados Unidos, por la baja fortaleza en el enlace. Se aconseja una mirada especial en Alemania, porque si bien podría creerse que la cantidad mayor de citaciones obedece a una mayor antigüedad de las publicaciones, esto no es así, ya que las publicaciones de este país son por el contrario las más recientes y por tanto con mayor impacto.

\section{- Respecto al objetivo general 3}

Sí existe impacto de la reducción de cantidad de documentos en 1 y aumento en la cantidad de citaciones por autor en 4 como parámetros de filtro del tema de investigación sobre el ratio citaciones/documentos por autor, ya que para el primer escenario de 2 documentos como mínimo y 1 citación como mínimo se obtuvo que sería relevante detenerse en los documentos que pertenecen a los autores Dufour, Galante y Jones, mientras que con el segundo escenario de 1 documento como mínimo y 5 citaciones como mínimo, los autores que más deberían consultarse serían Hahne, Mccaleer y Mcconville por corresponderles el mayor ratio de citaciones/documentos.

Se recomienda centrarse en los autores Hahne, Mccaleer y Mcconville, porque el ratio citación /documento es 7,73 veces el número obtenido con los autores Dufour, Galante y Jones. Por otro lado, se recomienda a los autores Hahne, Mccaleer y Mcconville, porque sus publicaciones a pesar de ser del 2017, es decir solo 2 años antes que las de los autores Amanvermez, Bocking, Breedvelt, Karyotakie y Cuijpers, tienen una cantidad de citaciones de 5,27 veces la obtenida por estos últimos. Asimismo, aunque a los autores Cuijpers y Ebert según el primer escenario les corresponde publicaciones más recientes que Dufour, Galante y Jones, los autores Hahne, Mccaleer y Mcconville deben seguirse priorizando por tener ratio citación/documento de 10,54 veces el número obtenido por Cuijpers y Ebert.

\section{Referencias}

Aguilera Eguía, R. (2014). ¿Revisión sistemática, revisión narrativa o metaanálisis? Revista de La Sociedad Española Del Dolor, 21(6), 359-360. https://doi.org/10.4321/S113480462014000600010 
Cadena Iñiguez, P., de la Cruz-Morales, F. Del, Rosario, Rendón-Medel, R., Aguilar-Ávila, J., SalinasCruz, Eileen, \& Sangerman-Jarquín, D. M. (2017). Métodos cuantitativos, métodos cualitativos o su combinación en la investigación: un acercamiento en las ciencias sociales. Revista Mexicana de Ciencias Agrícolas, 8(7), 17. http://www.scielo.org.mx/scielo.php?script=sci_arttext\&pid=S20070934201700070 1603

Cortés, J. (2015). Web of Science: termómetro de la producción internacional de conocimiento. Ventajas y limitaciones. CULCyT, 29, 5-15.

Cortés Cortés, M., \& Iglesias León, M. (2004). Generalidades sobre Metodologías de la Investigación. Universidad Autónoma Del Carmen, 1-105.

http://www.unacar.mx/contenido/gaceta/ediciones/metodologia_investigacion.pdf

Diseño de la investigación - Dialnet. (n.d.). Retrieved October 11, 2020, from https://dialnet.unirioja.es/servlet/articulo? codigo $=6201669$

Fernández, A. P., \& Díaz, P. (2003). La investigación cualitativa y la investigación cuantitativa. Investigación Educativa, 7(11), 72-91.

Hernández, R., Fernández, C. \& Baptista, P. (2014). Metodología de la investigación. McGraw-Hill Education.

Maegraith, B. (1968). Tropical diseases. 2. Malaria. (II). Management and treatment. In Nursing times (Vol. 64, Issue 12).

Martín, M. del C. P. (2015). Abducción, método científico e Historia. Un acercamiento al pensamiento de Charles Pierce. Revista Paginas, 7(14), 125. https://doi.org/10.35305/rp.v7i14.161 
Moñivas, A., García-Diex, G., \& García-de-Silva, R. (2012). Mindfulness (atención plena): concepto y teoría [ Mindfulness: Concept and Theory ]. Portularia, 12(Addenda), 83-89. https://doi.org/10.5218/prts.2012.0009

Muñiz, M. (1994). 1_Estudios-De-Caso-En-La-Investigación-Cualitativa. 1-8.

Paneque, R. J., \& Habana, L. (1998). Metodología De La Investigación Elementos Básicos Para La Investigación Clínica. 1-95.

Qualitative, T. H. E., \& In, I. (2006). La investigación cualitativa en educación. 10(18), 5766.

Redero-García, E. (2016). Aplicación del mindfulness en el proceso de enseñanzaaprendizaje en Educación Primaria. http://reunir.unir.net/handle/123456789/3945

Revista, A. L. A., Salud, E. D. E., \& Gálvez, C. (2016). Visualización De Las Principales Líneas De Investigación En Salud Pública: Un Análisis Basado En Mapas Bibliométricos Aplicados a La Revista Española De Salud Pública (2006-2015). Revista Española de Salud Pública, 90, 1-10.

Ritchie, A., Teufel, S., \& Robertson, S. (2008). Using terms from citations for IR: Some first results. Lecture Notes in Computer Science (Including Subseries Lecture Notes in Artificial Intelligence and Lecture Notes in Bioinformatics), 4956 LNCS(April), 211-221. https://doi.org/10.1007/978-3-54078646-7_21

Rodríguez Otero, M. C. (2015). Manual de mendeley GOOGLE. http://biblioteca.ucm.es/data/cont/docs/397-2013-12-12-guiadeusodemendeley2.pdf

Sabour, S. (2017). Reliability of smartphone-based teleradiology for evaluating thoracolumbar spine fractures: statistical issue to avoid misinterpretation. Spine Journal, 17(8), 1200. https://doi.org/10.1016/j.spinee.2017.04.017

Salgado, C. (2018). Teoría y práctica para hacer la tesis según la metodología cuantitativa. 
Scopus - Research Intelligence. (2019). 1-83.

Tesauro de la UNESCO. (12 de octubre de 2020). https://vocabularies.unesco.org.

Urrutia, G., \& Bonfill, X. (2010). PRISMA_Spanish.pdf. In Medicina Clínica (Vol. 135, Issue 11, pp. 507-511). http://es.cochrane.org/sites/es.cochrane.org/files/public/uploads/PRISMA_Spanish.pd $\mathrm{f}$

Vargas Cordero, Z. R. (2009). La Investigación aplicada: Una forma de conocer las realidades con evidencia científica. Revista Educación, 33(1), 155. https://doi.org/10.15517/revedu.v33i1.538 